\title{
AUTORESONANCE IN OSCILLATING SYSTEMS WITH COMBINED EXCITATION AND WEAK DISSIPATION
}

\author{
OSKAR A. SULTANOV \\ Institute of Mathematics, Ufa Federal Research Center, Russian Academy of Sciences, 112, \\ Chernyshevsky str., Ufa 450008 Russia.
}

\begin{abstract}
A mathematical model describing the initial stage of the capture into autoresonance for nonlinear oscillating systems with combined parametric and external excitation is considered. The solutions with unboundedly growing amplitude and limited phase mismatch correspond to the autoresonant capture. The paper investigates the existence, stability and bifurcations of such solutions in the presence of a weak dissipation in the system. Our technique is based on the study of particular solutions with power-law asymptotics at infinity and the construction of suitable Lyapunov functions.
\end{abstract}

Keywords: nonlinear oscillations, autoresonance, stability, Lyapunov function

Mathematics Subject Classification: 34C15, 34D05, 37B25, 37B55, 93D20

\section{INTRODUCTION}

Autoresonance is a phenomenon that occurs in nonlinear systems with slowly varying oscillating perturbations. Under certain conditions, the system automatically adjusts to the disturbances and holds this state for a sufficiently long period of time. As a result, the energy of the system can increase significantly [1. The autoresonance was first studied in the problems associated with the acceleration of particles [2,3] and planetary dynamics [4,5. Nowadays, it is considered as a universal phenomenon with a wide range of applications [6-13. The study of the corresponding mathematical models leads to new and challenging problems in the field of nonlinear dynamics [14 16].

Mathematical models associated with the autoresonance have been studied in many papers. See, for instance, [17 21], where the systems with external driving were analyzed, and [22 26], where the models of parametric autoresonance were investigated. The effect of a combined external and parametric excitation on the autoresonant capture in nonlinear systems was first studied in [27,28. In this paper, the autoresonance model with the combined excitation in the presence of a weak dissipation is considered, and the existence and stability of different autoresonant modes are discussed.

The paper is organized as follows. In section 1, the mathematical formulation of the problem is given. In section 2, the particular autoresonant solutions are described and the partition of a parameter space is constructed. The stability of particular solutions and asymptotics for general autoresonant solutions are discussed in section 3. A discussion of the results obtained is contained in section 4 .

\section{Problem statement}

Consider the non-autonomous system of two differential equations:

$$
\begin{aligned}
\frac{d \rho}{d \tau}+\gamma(\tau) \rho & =\alpha(\tau) \sin \psi-\beta(\tau) \rho \sin (2 \psi+\nu), \\
\rho\left[\frac{d \psi}{d \tau}-\rho^{2}+\lambda \tau\right] & =\alpha(\tau) \cos \psi-\beta(\tau) \rho \cos (2 \psi+\nu),
\end{aligned}
$$

with the parameters $\lambda \neq 0$ and $\nu \in[0, \pi)$. Smooth given functions $\alpha(\tau) \not \equiv 0$ and $\beta(\tau)$ correspond to the amplitude of an external and a parametric driving, a positive function $\gamma(\tau)$ is associated

E-mail address: oasultanov@gmail.com. 
with a dissipation. This system arises in the study of the autoresonance phenomena in a class of nonlinear oscillatory systems with a combined chirped-frequency excitation and a weak dissipation. The functions $\rho(\tau), \psi(\tau)$ describe the evolution of the amplitude and the phase mismatch of the oscillators. The solutions with $\rho(\tau) \sim \sqrt{\lambda \tau}$ and $\psi(\tau) \sim \sigma, \sigma=$ const as $\tau \rightarrow \infty$ are associated with the phase-locking phenomenon and the capture into autoresonance. Note that system (11) also has non-autoresonant solutions with a bounded amplitude, but such solutions are not considered in the present paper.

The combined effect of parametric and external excitations is determined by the behaviour of the ratio $f(\tau) \equiv \beta(\tau) / \alpha(\tau)$ as $\tau \rightarrow \infty$. Indeed, if $f(\tau) \sim f_{0} \tau^{-1 / 2-\varkappa}, \varkappa>0, f_{0}=$ const $\neq 0$, the parametric pumping is insignificant and system (11) corresponds to a perturbation of the model with the external driving. If $f(\tau) \sim f_{0} \tau^{-1 / 2+\varkappa}$, the impact of external driving becomes inconsiderable and the system takes the form of a perturbed model of parametric autoresonance. The parametric and external excitations are comparable when $f(\tau) \sim f_{0} \tau^{-1 / 2}$. Note also that the existence of autoresonant solutions in systems with a dissipation depends on the behaviour of the function $g(\tau) \equiv \gamma(\tau) / \alpha(\tau)$ as $\tau \rightarrow \infty$. From the first equation in (11) it follows that the necessary condition is $g(\tau) \sim g_{0} \tau^{-1 / 2-\varkappa}$ with $\varkappa \geq 0, g_{0}=$ const $\neq 0$. Thus, in this paper it is assumed that

$$
\alpha(\tau)=\tau^{\frac{1}{2}} \sum_{k=0}^{\infty} \alpha_{k} \tau^{-k}, \quad \beta(\tau)=\sum_{k=0}^{\infty} \beta_{k} \tau^{-k}, \quad \gamma(\tau)=\sum_{k=0}^{\infty} \gamma_{k} \tau^{-k}, \quad \tau \rightarrow \infty, \quad \alpha_{k}, \beta_{k}, \gamma_{k}=\text { const. }
$$

Without loss of generality, we assume that $\alpha_{0}=1$ and $\gamma_{0} \neq 0$.

Note that system (11) appears after averaging of perturbed oscillatory nonlinear systems and describes a long term evolution of solutions. For a system with one degree of freedom, the example is given by the following equation:

$$
\frac{d^{2} x}{d t^{2}}+\epsilon C(\epsilon t) \frac{d x}{d t}+(1+\epsilon B(\epsilon t) \cos (2 \zeta(t)-\nu)) U^{\prime}(x)=\epsilon A(\epsilon t) \cos \zeta(t),
$$

where $\zeta(t)=t-\vartheta t^{2}, U(x)=x^{2} / 2-\epsilon x^{4} / 24+\mathcal{O}\left(\epsilon^{2}\right), 0<\epsilon, \vartheta \ll 1$. We see that equation (2) with $\epsilon=0$ has a stable trivial solution $x(t) \equiv 0, \dot{x}(t) \equiv 0$. Solutions of the perturbed equation with small enough initial data $\left(x(0), x^{\prime}(0)\right)$, whose the energy $E(t) \equiv U(x(t))+\left(x^{\prime}(t)\right)^{2} / 2$ increases significantly with time and the phase $\Phi(t)$ is synchronised with the pumping such that $\Phi(t)-\zeta(t)=\mathcal{O}(1)$, correspond to the capture into autoresonance. The approximation of such solutions is constructed by using the method of two scales with slow and fast variables: $\tau=\epsilon t / 4$ and $\zeta=\zeta(t)$. The substitution

$$
x(t)=2 \rho(\tau) \cos (\zeta+\psi(\tau))+\mathcal{O}(\epsilon)
$$

into equation (2) and the averaging over the fast variable lead to system (11) for the slowly varying functions $\rho(\tau)$ and $\psi(\tau)$ with $\lambda=16 \vartheta \epsilon^{-2}, \alpha(\tau)=A(\epsilon t), \beta(\tau)=B(\epsilon t), \gamma(\tau)=2 C(\epsilon t)$. Likewise, system (11) is derived in many other nonlinear problems related to autoresonance, including infinitedimensional systems (see [15].

In this paper, the conditions for the existence and stability of autoresonant solutions to system (11) are discussed. Our technique is based on the analysis of particular solutions with power-law asymptotics at infinity. In the first step, such solutions are constructed and the conditions for their existence specify the partition of the parameter space. Then, the Lyapunov stability of the particular solutions is investigated. Since the considered system is non-autonomous, the use of linear stability analysis is limited and nonlinear terms of equations must be taken into account. In this case, the stability can be justified with the Lyapunov function method. The presence of stability will ensure the existence of a family of autoresonant solutions. For such solutions, the asymptotic estimates at infinity are obtained at the last step from the properties of the constructed Lyapunov functions.

\section{Particular autoresonant solutions}

Consider the particular autoresonant solutions having the following asymptotics:

$$
\rho_{*}(\tau)=\rho_{-1} \sqrt{\tau}+\rho_{0}+\sum_{k=1}^{\infty} \rho_{k} \tau^{-\frac{k}{2}}, \quad \psi_{*}(\tau)=\psi_{0}+\sum_{k=1}^{\infty} \psi_{k} \tau^{-\frac{k}{2}}, \quad \tau \rightarrow \infty .
$$


Substituting these series into system (11) and grouping the terms of the same power of $\tau$ yield $\rho_{-1}=\sqrt{\lambda}$, $\rho_{0}=0$, and $\psi_{0}=\sigma$, where $\sigma$ satisfies the equation

$$
\mathcal{P}(\sigma ; \delta, \nu, \kappa) \equiv \delta \sin (2 \sigma+\nu)-\sin \sigma+\kappa=0, \quad \delta=\beta_{0} \sqrt{\lambda}, \quad \kappa=\gamma_{0} \sqrt{\lambda}
$$

Note that the number of roots to equation depends on the values of the parameters $(\delta, \nu, \kappa)$. If, in addition, the inequality $\mathcal{P}^{\prime}(\sigma ; \delta, \nu, \kappa) \neq 0$ holds, the remaining coefficients $\rho_{k}, \psi_{k}$ as $k \geq 1$ are determined from the chain of linear equations:

$$
\begin{aligned}
2 \sqrt{\lambda} \rho_{k} & =\mathcal{A}_{k}\left(\rho_{-1}, \ldots, \rho_{k-1}, \sigma, \psi_{1}, \ldots, \psi_{k-1}\right), \\
\mathcal{P}^{\prime}(\sigma ; \delta, \nu, \kappa) \psi_{k} & =\mathcal{B}_{k}\left(\rho_{-1}, \ldots, \rho_{k-1}, \sigma, \psi_{1}, \ldots, \psi_{k-1}\right),
\end{aligned}
$$

where

$$
\begin{aligned}
\mathcal{A}_{1}= & \frac{1}{\sqrt{\lambda}}(\delta \cos (2 \sigma+\nu)-\cos \sigma), \\
\mathcal{A}_{2}= & -\frac{\psi_{1}}{\sqrt{\lambda}}(2 \delta \sin (2 \sigma+\nu)-\sin \sigma), \\
\mathcal{A}_{3}= & -\rho_{1}^{2}+\beta_{1} \cos (2 \sigma+\nu)-\left(\alpha_{1}-\frac{\rho_{1}}{\sqrt{\lambda}}\right) \frac{\cos \sigma}{\sqrt{\lambda}} \\
& -\frac{\psi_{2}}{\sqrt{\lambda}}(2 \delta \sin (2 \sigma+\nu)-\sin \sigma)-\frac{\psi_{1}^{2}}{2 \sqrt{\lambda}}(4 \delta \cos (2 \sigma+\nu)-\cos \sigma), \\
\mathcal{B}_{1}= & 0, \\
\mathcal{B}_{2}= & -\mathcal{P}^{\prime \prime}(\sigma ; \delta, \nu, \kappa) \frac{\psi_{1}^{2}}{2}+\left(\alpha_{1}-\frac{\rho_{1}}{\sqrt{\lambda}}\right) \sin \sigma-\beta_{1} \sqrt{\lambda} \sin (2 \sigma+\nu)-\left(1+2 \gamma_{1}\right) \frac{\rho_{-1}}{2}, \\
\mathcal{B}_{3}= & -\psi_{1} \psi_{2} \mathcal{P}^{\prime \prime}(\sigma ; \delta, \nu, \kappa)-\frac{\rho_{2}}{\sqrt{\lambda}} \sin \sigma-\frac{\psi_{1}^{3}}{6} \mathcal{P}^{\prime \prime \prime}(\sigma ; \delta, \nu, \kappa)+\alpha_{1} \psi_{1} \cos \sigma \\
& -2 \psi_{1}\left(\beta_{1} \sqrt{\lambda}+\beta_{0} \rho_{1}\right) \cos (2 \sigma+\nu),
\end{aligned}
$$

etc. In particular,

$$
\psi_{1}=0, \quad \psi_{2}=\theta, \quad \theta:=\frac{\mathcal{B}_{2}(\sigma)}{\mathcal{P}^{\prime}(\sigma ; \delta, \nu, \kappa)} .
$$

Note that the pair of equations $\mathcal{P}(\sigma ; \delta, \nu, \kappa)=0$ and $\mathcal{P}^{\prime}(\sigma ; \delta, \nu, \kappa)=0$ defines a bifurcation surface $S=S_{1} \cup S_{2}$ in the parameter space $(\delta, \nu, \kappa)$, where

$$
\begin{gathered}
S_{j}:=\left\{(\delta, \nu, \kappa) \in \mathbb{R} \times[0, \pi) \times \mathbb{R}: \sin \nu=p_{j}(\delta, \kappa)\right\}, \\
p_{j}(\delta, \kappa):=\delta^{-1}\left(\kappa\left(2 \sin ^{2} \varsigma_{j}-1\right)-\sin ^{3} \varsigma_{j}\right), \quad \sin \varsigma_{j}=z_{j}(\delta, \kappa), \\
z_{j}(\delta, \kappa):=\frac{1}{3}\left(4 \kappa+(-1)^{j} \sqrt{4 \kappa^{2}+12 \delta^{2}-3}\right), \quad j \in\{1,2\} .
\end{gathered}
$$

For every $\kappa>0$, the bifurcation set is determined by the properties of $p_{1}(\delta, \kappa)$ and $p_{2}(\delta, \kappa)$ (see Fig. 1). In particular, if $0<\kappa<3 / 4$, there are two curves

$$
\begin{aligned}
& s_{+}:=\left\{(\delta, \nu) \in\left[n_{2}, m_{2}\right] \times[0, \pi): \sin \nu=p_{1}(\delta, \kappa)\right\}, \\
& s_{-}:=\left\{(\delta, \nu) \in\left[n_{1}, \delta_{*}\right] \times[0, \pi): \sin \nu=p_{1}(\delta, \kappa)\right\} \cup\left\{(\delta, \nu) \in\left[m_{1}, \delta_{*}\right] \times[0, \pi): \sin \nu=p_{2}(\delta, \kappa)\right\},
\end{aligned}
$$

dividing the parameter plane $(\delta, \nu)$ into tree parts (see Fig. 2,a):

$$
\begin{aligned}
\Omega_{+} & :=\left\{(\delta, \nu) \in \mathbb{R} \times[0, \pi): \delta>s_{+}\right\}, \quad \Omega_{-}:=\left\{(\delta, \nu) \in \mathbb{R} \times[0, \pi): \delta<s_{-}\right\}, \\
\Omega_{0} & :=\left\{(\delta, \nu) \in \mathbb{R} \times[0, \pi): s_{-}<\delta<s_{+}\right\},
\end{aligned}
$$

where $\delta_{*}=-\sqrt{\left(3-4 \kappa^{2}\right) / 12}, n_{1}<n_{2}$ are the roots of the equation $p_{1}(n, \kappa)=0, m_{1}=\kappa-1$, $m_{2}=\kappa+1$. In this case, the equation $\mathcal{P}(\sigma ; \delta, \nu, \kappa)=0$ has four different roots on the interval $[0,2 \pi)$ if $(\delta, \nu) \in \Omega_{-} \cup \Omega_{+}$. If $(\delta, \nu) \in \Omega_{0}$, there are only two different roots (see Fig. 3, a). 


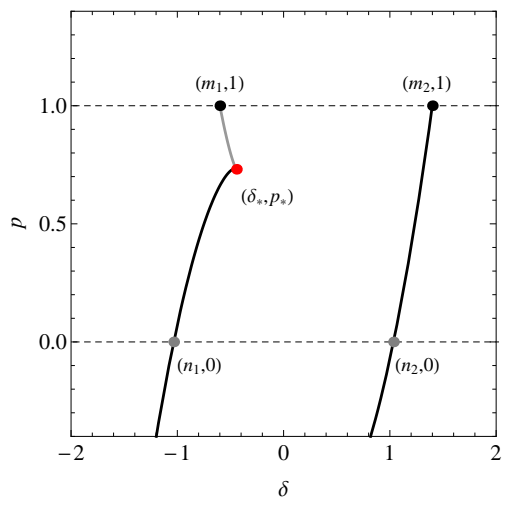

(a) $\kappa=0.4$

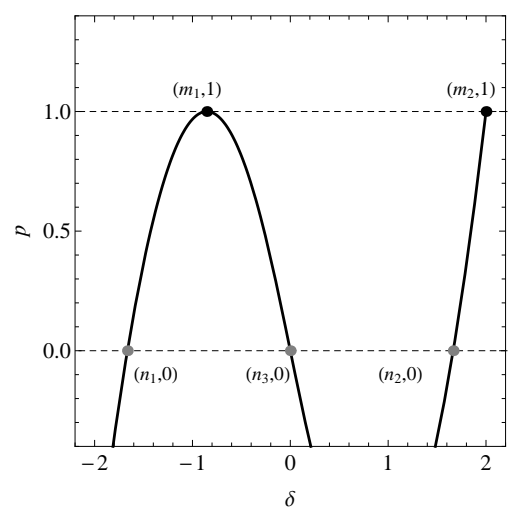

(c) $\kappa=1$

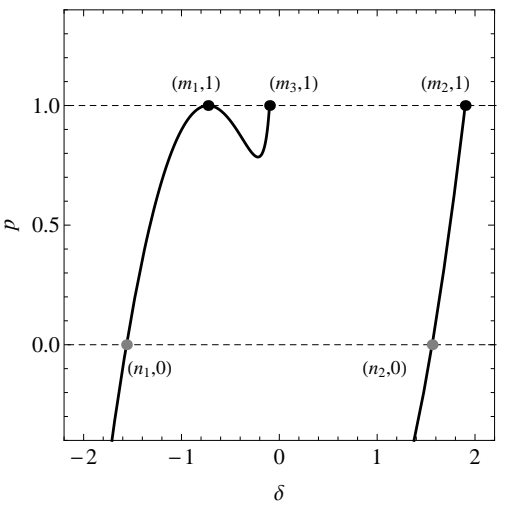

(b) $\kappa=0.9$

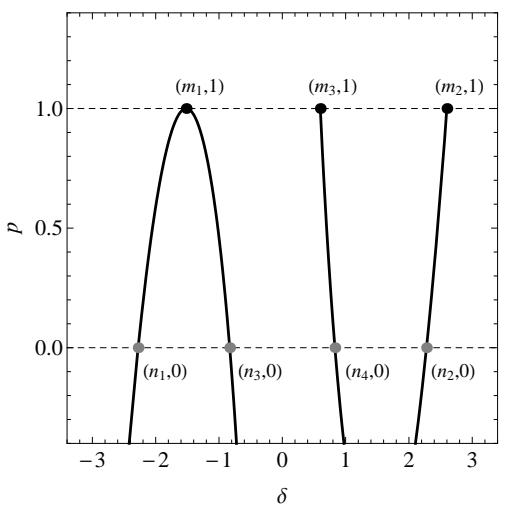

(d) $\kappa=1.6$

FiguRE 1. Graphs of $p_{1}(\delta, \kappa)$ (black curves) and $p_{2}(\delta, \kappa)$ (gray curves) as functions of the parameter $\delta$ with fixed $\kappa$.

If $3 / 4 \leq \kappa<1$, there are three curves

$$
\begin{aligned}
& s_{+}:=\left\{(\delta, \nu) \in\left[n_{2}, m_{2}\right] \times[0, \pi): \sin \nu=p_{1}(\delta, \kappa)\right\}, \\
& s_{-}:=\left\{(\delta, \nu) \in\left[n_{1}, m_{1}\right] \times[0, \pi): \sin \nu=p_{1}(\delta, \kappa)\right\}, \\
& s_{0}:=\left\{(\delta, \nu) \in\left[m_{1}, m_{3}\right] \times[0, \pi): \sin \nu=p_{1}(\delta, \kappa)\right\},
\end{aligned}
$$

dividing the parameter plane $(\delta, \nu)$ into four parts (see Fig. 2, b):

$$
\begin{aligned}
& \Omega_{+}:=\left\{(\delta, \nu) \in \mathbb{R} \times[0, \pi): \delta>s_{+}\right\}, \quad \Omega_{-}:=\left\{(\delta, \nu) \in \mathbb{R} \times[0, \pi): \delta<s_{-}\right\}, \\
& \Omega_{*}:=\left\{\delta \in\left[m_{1}, m_{3}\right], \arcsin p_{1}(\delta, \kappa)<\nu<\pi-\arcsin p_{1}(\delta, \kappa)\right\}, \\
& \Omega_{0}:=\mathbb{R} \times[0, \pi) \backslash \overline{\left(\Omega_{+} \cup \Omega_{-} \cup \Omega_{*}\right)},
\end{aligned}
$$

where $n_{1}<n_{2}$ are the roots of the equation $p_{1}(n, \kappa)=0, m_{1}=-\left(\sqrt{2} \kappa+\sqrt{2 \kappa^{2}-1}\right) / \sqrt{8}, m_{3}=\kappa-1$, $m_{2}=\kappa+1$ are the roots of the equation $p_{1}(m, \kappa)=1$.

If $\kappa=1$, the equation $p_{1}(\delta, \kappa)=0$ has three different roots $n_{1}<n_{3}<n_{2}$. In this case, the parameter plane $(\delta, \nu)$ is divided into the following parts (see Fig. 2, c):

$$
\begin{aligned}
& \Omega_{+}:=\left\{(\delta, \nu) \in \mathbb{R} \times[0, \pi): \delta>s_{+}\right\}, \quad \Omega_{-}:=\left\{(\delta, \nu) \in \mathbb{R} \times[0, \pi): \delta<s_{-}\right\}, \\
& \Omega_{*}:=\left\{\delta \in\left[m_{1}, n_{3}\right]: \arcsin p_{1}(\delta, \kappa)<\nu<\pi-\arcsin p_{1}(\delta, \kappa)\right\}, \\
& \Omega_{0}:=\mathbb{R} \times[0, \pi) \backslash \overline{\left(\Omega_{+} \cup \Omega_{-} \cup \Omega_{*}\right)},
\end{aligned}
$$

by the curves

$$
\begin{aligned}
s_{+}:=\left\{(\delta, \nu) \in\left[n_{2}, m_{2}\right] \times[0, \pi): \sin \nu=p_{1}(\delta, \kappa)\right\} \\
s_{-}:=\left\{(\delta, \nu) \in\left[n_{1}, m_{1}\right] \times[0, \pi): \sin \nu=p_{1}(\delta, \kappa)\right\} \\
s_{0}:=\left\{(\delta, \nu) \in\left[m_{1}, n_{3}\right] \times[0, \pi): \sin \nu=p_{1}(\delta, \kappa)\right\} \cup\{\delta=0, \nu \in[0, \pi)\}
\end{aligned}
$$




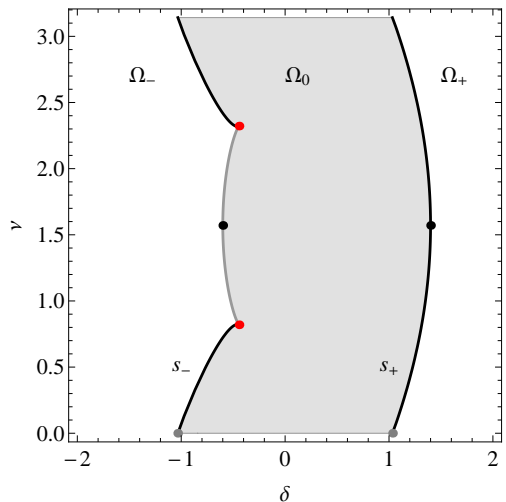

(a) $\kappa=0.4$

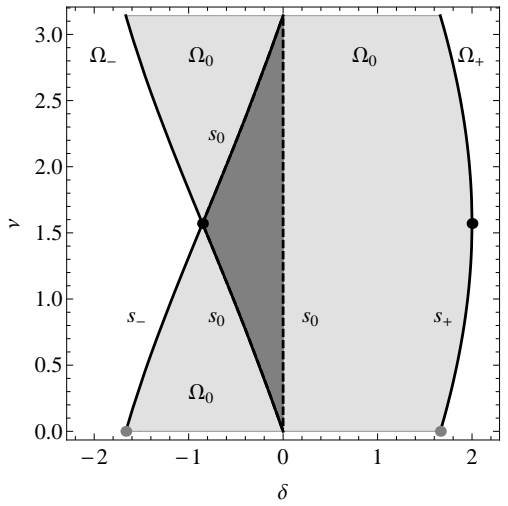

(c) $\kappa=1$

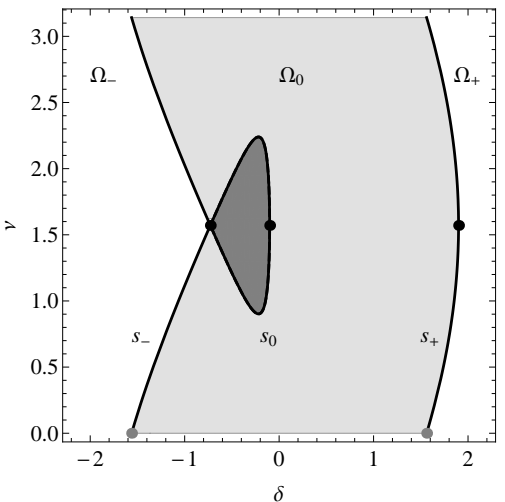

(b) $\kappa=0.9$

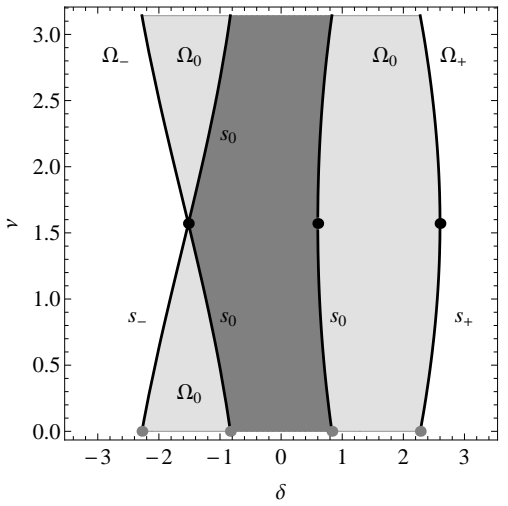

(d) $\kappa=1.6$

Figure 2. Partition of the parameter plane $(\delta, \nu)$.

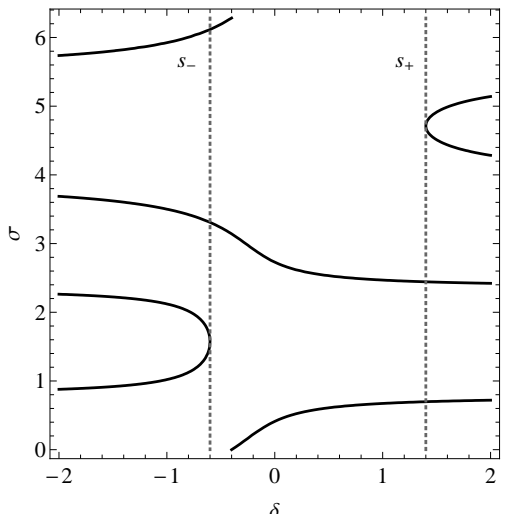

(a) $\kappa=0.4, \nu=\frac{\pi}{2}$

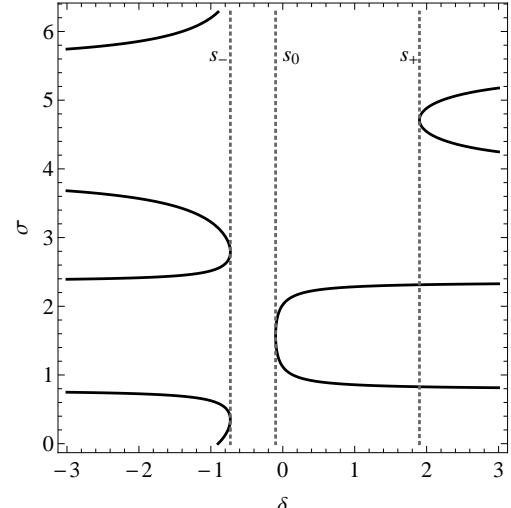

(b) $\kappa=0.9, \nu=\frac{\pi}{2}$

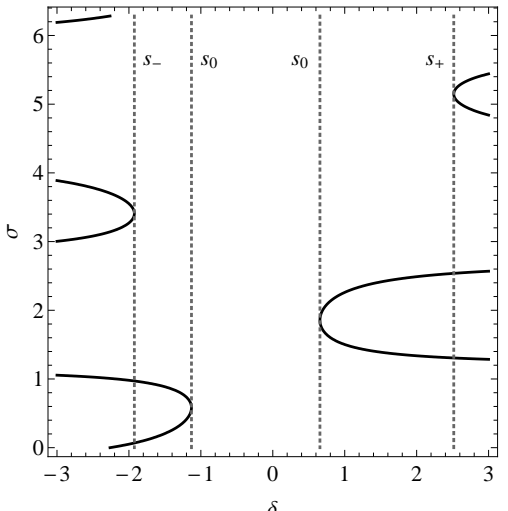

(c) $\kappa=1.6, \nu=\frac{\pi}{4}$

FiguRE 3. The roots to equation (4) as functions of the parameter $\delta$. The vertical dotted lines correspond to $s_{-}, s_{0}$ and $s_{+}$.

where $m_{1}=-(1+\sqrt{2}) / \sqrt{8}, m_{2}=2, p_{1}\left(m_{1,2}, \kappa\right) \equiv 1$.

If $\kappa>1$, the equation $p_{1}(\delta, \kappa)=0$ has four different roots $n_{1}<n_{3}<n_{4}<n_{2}$ and the parameter plane $(\delta, \nu)$ is divided by the curves

$$
\begin{aligned}
& s_{+}:=\left\{(\delta, \nu) \in\left[n_{2}, m_{2}\right] \times[0, \pi): \sin \nu=p_{1}(\delta, \kappa)\right\}, \\
& s_{-}:=\left\{(\delta, \nu) \in\left[n_{1}, m_{1}\right] \times[0, \pi): \sin \nu=p_{1}(\delta, \kappa)\right\}, \\
& s_{0}:=\left\{\delta \in\left[m_{1}, n_{3}\right] \cup\left[m_{3}, n_{4}\right], \nu \in[0, \pi): \sin \nu=p_{1}(\delta, \kappa)\right\}
\end{aligned}
$$




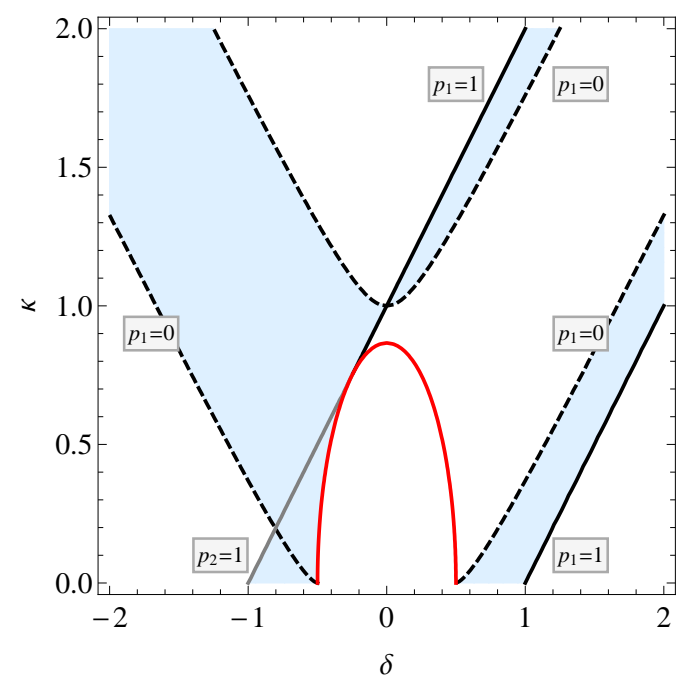

FIGURE 4. Existence domain (shaded area) of multiple roots to equation (4).

into four parts (see Fig. 2, d):

$$
\begin{aligned}
\Omega_{+}:= & \left\{(\delta, \nu) \in \mathbb{R} \times[0, \pi): \delta>s_{+}\right\}, \quad \Omega_{-}:=\left\{(\delta, \nu) \in \mathbb{R} \times[0, \pi): \delta<s_{-}\right\}, \\
\Omega_{*}:= & \left\{\delta \in\left[m_{1}, n_{3}\right], \arcsin p_{1}(\delta, \kappa)<\nu<\pi-\arcsin p_{1}(\delta, \kappa)\right\} \cup\left\{(\delta, \nu) \in\left[n_{3}, m_{3}\right] \times[0, \pi)\right\} \\
& \cup\left\{\delta \in\left[m_{3}, n_{4}\right], 0 \leq \nu<\arcsin p_{1}(\delta, \kappa)\right\} \cup\left\{\delta \in\left[m_{3}, n_{4}\right], \pi-\arcsin p_{1}(\delta, \kappa)<\nu<\pi\right\}, \\
\Omega_{0}:= & \mathbb{R} \times[0, \pi) \backslash \overline{\left(\Omega_{+} \cup \Omega_{-} \cup \Omega_{*}\right)},
\end{aligned}
$$

where $m_{1}=-\left(\sqrt{2} \kappa+\sqrt{2 \kappa^{2}-1}\right) / \sqrt{8}, m_{2}=\kappa+1, p_{1}\left(m_{1,2}, \kappa\right) \equiv 1$.

As above, if $(\delta, \nu) \in \Omega_{-} \cup \Omega_{+}$, the equation $\mathcal{P}(\sigma ; \delta, \nu, \kappa)=0$ has four different roots on the interval $[0,2 \pi)$. If $(\delta, \nu) \in \Omega_{0}$, there are only two different roots. If $(\delta, \nu) \in \Omega_{*}$, the equation has no solutions (see Fig. 3, b,c,d).

Thus we have

Theorem 1. If $(\delta, \nu) \in \Omega_{+} \cup \Omega_{-}$and $\kappa>0$, system (1) has four different solutions with asymptotic expansion in the form of a series (3). If $\kappa>0$ and $(\delta, \nu) \in \Omega_{0}$, system (11) has 2 different solutions with asymptotic expansion in the form of a series (3).

The existence of solutions $\rho_{*}(\tau), \psi_{*}(\tau)$ with the asymptotics (3) as $\tau \geq \tau_{*}$ follows from [29,30]. The comparison theorems [31] applied to system (11) guarantees that the solutions can be extended to the semi-axis.

2.1. The roots of multiplicity 2. If $(\delta, \nu) \in s_{-} \cup s_{+} \cup s_{0}$, there exists $\sigma$ such that $\mathcal{P}(\sigma ; \delta, \nu, \kappa)=0$ and $\mathcal{P}^{\prime}(\sigma ; \delta, \nu, \kappa)=0$. It can easily be checked that $\sigma \in\left\{\varsigma: \sin \varsigma=z_{1,2}(\delta, \kappa)\right\}$. The multiple roots exist if $(\delta, \kappa) \in \mathfrak{D}_{m}$, where $\mathfrak{D}_{m}:=\left(\left\{0 \leq p_{1}(\delta, \kappa) \leq 1\right\} \cup\left\{0 \leq p_{2}(\delta, \kappa) \leq 1\right\}\right) \cap\left(\left\{\left|z_{1}(\delta, \kappa)\right| \leq 1\right\} \cup\left\{\left|z_{2}(\delta, \kappa)\right| \leq\right.\right.$ $1\}) \cap\left\{\kappa^{2}+3 \delta^{2} \geq 3 / 4\right\}$ (see Fig. (4). In addition, suppose that $\mathcal{P}^{\prime \prime}(\sigma ; \delta, \nu, \kappa) \equiv-3 \sin \sigma+4 \kappa \neq 0$, then $\sigma$ is the root of multiplicity 2 . In this case, $\psi_{1}$ is determined from the equation:

$$
\begin{gathered}
\mathcal{P}^{\prime \prime}(\sigma ; \delta, \nu, \kappa) \frac{\psi_{1}^{2}}{2}=\mathcal{C}(\sigma), \\
\mathcal{C}(\sigma) \equiv \frac{\sin 2 \sigma-4 \lambda^{2}}{8 \lambda \sqrt{\lambda}}-\left(\beta_{1} \sqrt{\lambda} \sin (2 \sigma+\nu)-\alpha_{1} \sin \sigma+\gamma_{1} \sqrt{\lambda}\right) .
\end{gathered}
$$

It follows that the asymptotic solution in the form (3) does not exist when $\mathcal{P}^{\prime \prime}(\sigma ; \delta, \nu, \kappa) \mathcal{C}(\sigma)<0$. If $\mathcal{P}^{\prime \prime}(\sigma ; \delta, \nu, \kappa) \mathcal{C}(\sigma)>0$, equation (5) has two different roots:

$$
\psi_{1}= \pm \phi, \quad \phi:=\sqrt{\frac{2 \mathcal{C}(\sigma)}{\mathcal{P}^{\prime \prime}(\sigma ; \delta, \nu, \kappa)}} .
$$


Note that if $\alpha_{1}=\beta_{1}=\gamma_{1}=0$ and $\lambda>1 / 2$, then $\mathcal{C}(\sigma)<0$ for all $\sigma \in \mathbb{R}$ and a suitable root is $\sigma$ such that $\sin \sigma=z_{1}(\delta, \kappa)$. The remaining coefficients $\rho_{k}, \psi_{k}$ are determined from the following recurrent system of equations:

$$
\begin{aligned}
2 \sqrt{\lambda} \rho_{k} & =\mathcal{A}_{k}\left(\rho_{-1}, \ldots, \rho_{k-1}, \sigma, \psi_{1}, \ldots, \psi_{k-1}\right), \\
\mathcal{P}^{\prime \prime}(\sigma ; \delta, \nu, \kappa) \psi_{1} \psi_{k}+\frac{\sin \sigma}{\sqrt{\lambda}} \rho_{k} & =\mathcal{C}_{k}\left(\rho_{-1}, \ldots, \rho_{k-1}, \sigma, \psi_{1}, \ldots, \psi_{k-1}\right),
\end{aligned}
$$

where

$$
\begin{aligned}
\mathcal{C}_{2}= & -\frac{\psi_{1}^{3}}{6} \mathcal{P}^{\prime \prime \prime}(\sigma ; \delta, \nu, \kappa)+\alpha_{1} \psi_{1} \cos \sigma-2 \psi_{1}\left(\beta_{1} \sqrt{\lambda}+\beta_{0} \rho_{1}\right) \cos (2 \sigma+\nu), \\
\mathcal{C}_{3}= & \frac{\rho_{1}}{2}-\gamma_{1} \rho_{1}-\gamma_{2} \sqrt{\lambda}-\frac{\psi_{2}^{2}}{2} \mathcal{P}^{\prime \prime}(\sigma ; \delta, \nu, \kappa)-\frac{\psi_{1}^{2} \psi_{2}}{2} \mathcal{P}^{\prime \prime \prime}(\sigma ; \delta, \nu, \kappa)-\frac{\psi_{1}^{4}}{24} \mathcal{P}^{(4)}(\sigma ; \delta, \nu, \kappa) \\
& -\alpha_{1}\left(\psi_{2} \cos \sigma-\frac{\psi_{1}^{2} \sin \sigma}{2}\right)-\left(2 \psi_{2}\left(\beta_{1} \sqrt{\lambda}+\beta_{0} \rho_{1}\right)+2 \psi_{1} \beta_{0} \rho_{2}\right) \cos (2 \sigma+\nu) \\
& +\left(2 \psi_{1}^{2}\left(\beta_{1} \sqrt{\lambda}+\beta_{0} \rho_{1}\right)+\alpha_{2}-\beta_{2} \sqrt{\lambda}-\beta_{1} \rho_{1}\right) \sin (2 \sigma+\nu),
\end{aligned}
$$

etc.

2.2. The roots of multiplicity 3. Now suppose $(\delta, \nu) \in s_{-} \cup s_{+} \cup s_{0}$ and $\sigma$ is the root of equation (41) such that $\mathcal{P}^{\prime}(\sigma ; \delta, \nu, \kappa)=0$ and $\mathcal{P}^{\prime \prime}(\sigma ; \delta, \nu, \kappa)=0$. These imply that $\mathcal{P}^{\prime \prime \prime}(\sigma ; \delta, \nu, \kappa) \equiv-3 \cos \sigma$, $\sigma \in\{\varsigma: \sin \varsigma=4 \kappa / 3\}, \kappa^{2}+3 \delta^{2}=3 / 4$, and $\sin \nu=-\kappa \delta^{-1}\left(1+32 \delta^{2}\right) / 9$. Let $\mathcal{P}^{\prime \prime \prime}(\sigma ; \delta, \nu, \kappa) \neq 0$. In this case, the asymptotic solutions are constructed in the following form:

$$
\rho(\tau)=\rho_{-1} \sqrt{\tau}+\rho_{0}+\sum_{k=1}^{\infty} \rho_{k} \tau^{-\frac{k}{6}}, \quad \psi(\tau)=\sigma+\sum_{k=1}^{\infty} \psi_{k} \tau^{-\frac{k}{6}}, \quad \tau \rightarrow \infty .
$$

It can easily be checked that

$$
\begin{gathered}
\rho_{-1}=\sqrt{\lambda}, \quad \rho_{0}=\rho_{1}=\rho_{2}=0, \quad \rho_{3}=-\frac{\cos \sigma}{4 \lambda}, \quad \psi_{1}=0, \quad \psi_{2}=\chi, \\
\chi:=\left[\frac{\mathcal{N}(\sigma)}{\mathcal{P}^{\prime \prime \prime}(\sigma ; \delta, \nu, \kappa)}\right]^{\frac{1}{3}}, \quad \mathcal{N}(\sigma) \equiv \frac{3\left(\sin 2 \sigma-4 \lambda^{2}\right)}{4 \lambda \sqrt{\lambda}}-6\left(\beta_{1} \sqrt{\lambda} \sin (2 \sigma+\nu)-\alpha_{1} \sin \sigma+\gamma_{1} \sqrt{\lambda}\right) .
\end{gathered}
$$

The remaining coefficient $\rho_{k+1}, \psi_{k}$ as $k \geq 3$ are determined from the following chain of equations:

$$
\begin{aligned}
2 \sqrt{\lambda} \rho_{k+1} & =\mathcal{M}_{k+1}\left(\rho_{-1}, \ldots, \rho_{k}, \sigma, \psi_{1}, \ldots, \psi_{k-1}\right), \\
\mathcal{P}^{\prime \prime \prime}(\sigma ; \delta, \nu) \frac{\psi_{2}^{2} \psi_{k}}{2}+\frac{\sin \sigma}{\sqrt{\lambda}} \rho_{k+1} & =\mathcal{N}_{k}\left(\rho_{-1}, \ldots, \rho_{k}, \sigma, \psi_{1}, \ldots, \psi_{k-1}\right),
\end{aligned}
$$

where

$$
\begin{aligned}
& \mathcal{M}_{4}=0, \quad \mathcal{M}_{5}=\frac{\psi_{2} \sin \sigma}{2 \sqrt{\lambda}}, \quad \mathcal{M}_{6}=\frac{\psi_{3} \sin \sigma}{2 \sqrt{\lambda}}, \quad \mathcal{M}_{7}=\frac{\psi_{4} \sin \sigma-\psi_{2}^{2} \cos \sigma}{2 \sqrt{\lambda}}, \\
& \mathcal{N}_{3}=0, \quad \mathcal{N}_{4}=-\psi_{2}\left(\beta_{1} \sqrt{\lambda}+\beta_{0} \rho_{3}-\alpha_{1}\right) \cos \sigma-\mathcal{P}^{\prime \prime \prime}(\sigma ; \delta, \nu, \kappa) \frac{\psi_{2} \psi_{3}^{2}}{2}-\mathcal{P}^{(4)}(\sigma ; \delta, \nu, \kappa) \frac{\psi_{2}^{4}}{24}, \\
& \mathcal{N}_{5}=-\psi_{3}\left(\beta_{1} \sqrt{\lambda}+\beta_{0} \rho_{3}-\alpha_{1}\right) \cos \sigma-\mathcal{P}^{\prime \prime \prime}(\sigma ; \delta, \nu, \kappa) \frac{6 \psi_{2} \psi_{3} \psi_{4}+\psi_{3}^{3}}{6}-\mathcal{P}^{(4)}(\sigma ; \delta, \nu, \kappa) \frac{\psi_{2}^{3} \psi_{3}}{6},
\end{aligned}
$$

etc. It can easily be checked that this system is solvable whenever $\mathcal{N}(\sigma) \neq 0$. Note that $\mathcal{N}(\sigma) \neq 0$ for any $\sigma \in \mathbb{R}$ if $\alpha_{1}=\beta_{1}=\gamma_{1}=0$ and $\lambda>1 / 2$.

2.3. The roots of multiplicity 4. Let $(\delta, \nu) \in s_{-} \cup s_{+} \cup s_{0}$ and $\sigma$ be the root of equation (44) such that $\mathcal{P}^{\prime}(\sigma ; \delta, \nu, \kappa)=0, \mathcal{P}^{\prime \prime}(\sigma ; \delta, \nu, \kappa)=0$ and $\mathcal{P}^{\prime \prime \prime}(\sigma ; \delta, \nu, \kappa)=0$. In this case, $\kappa=3 / 4, \delta=-1 / 4$, $\nu=\pi / 2, \sigma=\pi / 2$ and $\mathcal{P}^{(4)}(\sigma ; \delta, \nu, \kappa)=3$. The asymptotic solutions are constructed in the following form:

$$
\rho(\tau)=\rho_{-1} \sqrt{\tau}+\rho_{0}+\sum_{k=1}^{\infty} \rho_{k} \tau^{-\frac{k}{4}}, \quad \psi(\tau)=\sigma+\sum_{k=1}^{\infty} \psi_{k} \tau^{-\frac{k}{4}}, \quad \tau \rightarrow \infty .
$$


It follows easily that

$$
\rho_{-1}=\sqrt{\lambda}, \quad \rho_{0}=\rho_{1}=\rho_{2}=0, \quad \psi_{1}^{4}=\mathcal{Q}, \quad \mathcal{Q} \equiv 8 \alpha_{1}+4 \sqrt{\lambda}\left(2 \beta_{1}-2 \gamma_{1}-1\right) .
$$

If $\mathcal{Q}>0$, the last equation has two real, distinct roots: $\psi_{1}= \pm \xi, \xi:=\mathcal{Q}^{1 / 4}$. The remaining coefficient $\rho_{k+1}, \psi_{k}$ as $k \geq 2$ are determined from the following chain of equations:

$$
\begin{aligned}
2 \sqrt{\lambda} \rho_{k+1} & =\mathcal{T}_{k+1}\left(\rho_{-1}, \ldots, \rho_{k}, \psi_{1}, \ldots, \psi_{k-1}\right), \\
\psi_{1}^{3} \psi_{k}+\frac{2}{\sqrt{\lambda}} \rho_{k+1} & =\mathcal{Q}_{k}\left(\rho_{-1}, \ldots, \rho_{k}, \psi_{1}, \ldots, \psi_{k-1}\right),
\end{aligned}
$$

where

$$
\begin{aligned}
& \mathcal{T}_{3}=\frac{\psi_{1}}{2 \sqrt{\lambda}}, \quad \mathcal{T}_{4}=\frac{\psi_{2}}{2 \sqrt{\lambda}}, \quad \mathcal{T}_{5}=\frac{\psi_{1}^{3}+3 \psi_{3}}{6 \sqrt{\lambda}}, \quad \mathcal{T}_{6}=\frac{\psi_{1}^{2} \psi_{2}+\psi_{4}-2 \sqrt{\lambda} \rho_{2}^{2}}{2 \sqrt{\lambda}}, \\
& \mathcal{Q}_{2}=0, \quad \mathcal{Q}_{3}=-\alpha_{1} \psi_{1}^{2}-4 \beta_{1} \sqrt{\lambda} \psi_{1}^{2}+\frac{\psi_{1}^{6}}{24}-\frac{3 \psi_{1}^{2} \psi_{2}^{2}}{2}+\frac{\psi_{1}^{2} \rho_{2}}{\sqrt{\lambda}} \\
& \mathcal{Q}_{4}=-2 \alpha_{1} \psi_{1} \psi_{2}-8 \beta_{1} \sqrt{\lambda} \psi_{1} \psi_{2}+\psi_{1}^{5} \psi_{2}-\psi_{1} \psi_{2}^{3}-3 \psi_{1}^{2} \psi_{2} \psi_{3}-\frac{2 \psi_{1} \psi_{2} \rho_{2}}{\sqrt{\lambda}}+\frac{\psi_{1}^{2} \rho_{3}}{\sqrt{\lambda}},
\end{aligned}
$$

etc. If $\mathcal{Q} \leq 0$, the asymptotic solution in the form (7) does not exists.

Thus we have the following

Theorem 2. Let $(\delta, \nu) \in s_{-} \cup s_{-} \cup s_{0}, \kappa>0$ and $\sigma$ be a root of equation (44).

- If $\mathcal{P}^{\prime}(\sigma ; \delta, \nu, \kappa) \neq 0$, then there exists a solution $\rho_{*}(\tau), \psi_{*}(\tau)$ with asymptotic expansion in the form (3) with $\psi_{0}=\sigma, \psi_{1}=0, \psi_{2}=\theta$.

- If $\mathcal{P}^{\prime}(\sigma ; \delta, \nu, \kappa)=0$ and $\mathcal{P}^{\prime \prime}(\sigma ; \delta, \nu, \kappa) \mathcal{C}(\sigma)<0$, then system (11) has two solutions $\rho_{*}(\tau), \psi_{*}(\tau)$ with asymptotic expansion in the form (3) with $\psi_{0}=\sigma, \psi_{1}= \pm \phi$.

- If $\mathcal{P}^{\prime}(\sigma ; \delta, \nu, \kappa)=0, \mathcal{P}^{\prime \prime}(\sigma ; \delta, \nu, \kappa)=0$ and $\mathcal{N}(\sigma) \neq 0$, then system (1) has solution $\rho_{*}(\tau), \psi_{*}(\tau)$ with asymptotic expansion in the form (6) with $\psi_{1}=0, \psi_{2}=\chi$.

- If $\mathcal{P}^{\prime}(\sigma ; \delta, \nu, \kappa)=0, \mathcal{P}^{\prime \prime}(\sigma ; \delta, \nu, \kappa)=0, \mathcal{P}^{\prime \prime \prime}(\sigma ; \delta, \nu, \kappa)=0$ and $\mathcal{Q}>0$, then system (1) has two solutions $\rho_{*}(\tau), \psi_{*}(\tau)$ with asymptotic expansion in the form (7) with $\psi_{1}= \pm \xi$.

The proof is the same as that of Theorem 1.

\section{Stability ANALYSis}

3.1. Linear analysis. Let $\rho_{*}(\tau), \psi_{*}(\tau)$ be one of the particular autoresonant solutions with asymptotics (3), (6) or (7). The substitution $\rho(\tau)=\rho_{*}(\tau)+R(\tau), \psi(\tau)=\psi_{*}(\tau)+\Psi(\tau)$ into (1) gives the following system with a fixed point at $(0,0)$ :

(8)

$$
\begin{aligned}
& \frac{d R}{d \tau}+\gamma(\tau) R=\alpha(\tau)\left(\sin \left(\psi_{*}+\Psi\right)-\sin \psi_{*}\right)-\beta(\tau)\left(\left(\rho_{*}+R\right) \sin \left(2 \psi_{*}+2 \Psi+\nu\right)-\rho_{*} \sin \left(2 \psi_{*}+\nu\right)\right) \\
& \frac{d \Psi}{d \tau}=2 \rho_{*} R+R^{2}+\alpha(\tau)\left(\frac{\cos \left(\psi_{*}+\Psi\right)}{\rho_{*}+R}-\frac{\cos \psi_{*}}{\rho_{*}}\right)-\beta(\tau)\left(\cos \left(2 \psi_{*}+2 \Psi+\nu\right)-\cos \left(2 \psi_{*}+\nu\right)\right) .
\end{aligned}
$$

Consider the linearized system:

$$
\frac{d}{d \tau}\left(\begin{array}{l}
R \\
\Psi
\end{array}\right)=\boldsymbol{\Lambda}(\tau)\left(\begin{array}{l}
R \\
\Psi
\end{array}\right), \quad \boldsymbol{\Lambda}(\tau):=\left(\begin{array}{cc}
-\gamma(\tau)-\beta(\tau) \sin \left(2 \psi_{*}+\nu\right) & \alpha(\tau) \cos \psi_{*}-2 \beta(\tau) \rho_{*} \cos \left(2 \psi_{*}+\nu\right) \\
2 \rho_{*}-\frac{\alpha(\tau) \cos \psi_{*}}{\rho_{*}^{2}} & -\frac{\alpha(\tau) \sin \psi_{*}}{\rho_{*}}+2 \beta(\tau) \sin \left(2 \psi_{*}+\nu\right)
\end{array}\right)
$$

Define

$$
\hat{\rho}(\tau):=\frac{\rho_{*}(\tau)}{\sqrt{\lambda \tau}}-1, \quad \hat{\psi}(\tau):=\psi_{*}(\tau)-\sigma
$$

for $\tau \geq 0$, where $\sigma$ is one of the roots to equation (4). Then the functions $\hat{\rho}(\tau)$ and $\hat{\psi}(\tau)$ have the following asymptotics as $\tau \rightarrow \infty: \hat{\rho}(\tau)=\mathcal{O}\left(\tau^{-1}\right)$ and

- $\hat{\psi}(\tau)=\theta \tau^{-1}+\mathcal{O}\left(\tau^{-\frac{3}{2}}\right)$ if $\sigma$ is the simple root,

- $\hat{\psi}(\tau)= \pm \phi \tau^{-\frac{1}{2}}+\mathcal{O}\left(\tau^{-1}\right)$ if $\sigma$ is the root of multiplicity 2, 
- $\hat{\psi}(\tau)=\chi \tau^{-\frac{1}{3}}+\mathcal{O}\left(\tau^{-\frac{2}{3}}\right)$ if $\sigma$ is the root of multiplicity 3 ,

- $\hat{\psi}(\tau)= \pm \xi \tau^{-\frac{1}{4}}+\mathcal{O}\left(\tau^{-\frac{1}{2}}\right)$ if $\sigma$ is the root of multiplicity 4 .

Then the roots of the corresponding characteristic equation $|\boldsymbol{\Lambda}(\tau)-z \mathbf{I}|=0$ can be represented in the form

$$
z_{ \pm}(\tau)=\frac{1}{2}(\operatorname{tr} \boldsymbol{\Lambda}(\tau) \pm \sqrt{D(\tau)})
$$

where

$$
\begin{aligned}
\operatorname{tr} \boldsymbol{\Lambda}(\tau) & =\frac{1}{\sqrt{\lambda}}\left(-2 \kappa+\mathcal{P}\left(\psi_{*} ; \delta, \nu, \kappa\right)\right)+\mathcal{O}\left(\tau^{-1}\right) \\
D(\tau) & :=(\operatorname{tr} \boldsymbol{\Lambda}(\tau))^{2}-4 \operatorname{det} \boldsymbol{\Lambda}(\tau)=-8 \tau \sqrt{\lambda} \mathcal{P}^{\prime}\left(\psi_{*} ; \delta, \nu, \kappa\right)+\mathcal{O}(1)
\end{aligned}
$$

as $\tau \rightarrow \infty$. Therefore, if $\sigma$ is the simple root of (4) such that $\mathcal{P}^{\prime}(\sigma ; \delta, \nu, \kappa)<0$, then $z_{ \pm}(\tau)$ are real of different signs:

$$
z_{ \pm}(\tau)= \pm \tau^{\frac{1}{2}}(4 \lambda)^{\frac{1}{4}} \sqrt{-\mathcal{P}^{\prime}(\sigma ; \delta, \nu, \kappa)}+\mathcal{O}(1), \quad \tau \rightarrow \infty .
$$

This implies that the fixed point $(0,0)$ of $(8)$ is a saddle in the asymptotic limit, and the corresponding solutions $\rho_{*}(\tau), \psi_{*}(\tau)$ to system (11) are unstable (see, for example, [34).

Similarly, if $\sigma$ is the root of multiplicity 2 such that $\mp \mathcal{P}^{\prime \prime}(\sigma ; \delta, \nu, \kappa)>0$ and $\hat{\psi}(\tau)= \pm \phi \tau^{-1 / 2}+$ $\mathcal{O}\left(\tau^{-1}\right)$, then

$$
z_{ \pm}(\tau)= \pm \tau^{\frac{1}{4}}(4 \lambda)^{\frac{1}{4}} \sqrt{\left|\phi \mathcal{P}^{\prime \prime}(\sigma ; \delta, \nu, \kappa)\right|}+\mathcal{O}(1), \quad \tau \rightarrow \infty .
$$

In this case, the fixed point $(0,0)$ of $(8)$ and the corresponding particular solution to system (1) are both unstable.

In the same way, the fixed point of the linearized system is unstable when $\sigma$ is the root of multiplicity 3 and $\mathcal{P}^{\prime \prime \prime}(\sigma ; \delta, \nu)<0$. In this case, the eigenvalues have the following asymptotics:

$$
z_{ \pm}(\tau)= \pm \tau^{\frac{1}{6}} \lambda^{\frac{1}{4}} \sqrt{-\chi^{2} \mathcal{P}^{\prime \prime \prime}(\sigma ; \delta, \nu, \kappa)}+\mathcal{O}(1), \quad \tau \rightarrow \infty .
$$

If $\sigma$ is the root of multiplicity 4 and $\hat{\psi}(\tau)=-\xi \tau^{-1 / 4}+\mathcal{O}\left(\tau^{-1 / 2}\right)$, then

$$
z_{ \pm}(\tau)= \pm 2 \tau^{\frac{1}{8}} \lambda^{\frac{1}{4}} \xi^{\frac{3}{2}}+\mathcal{O}(1), \quad \tau \rightarrow \infty,
$$

and the corresponding particular solution to system (11) is unstable.

Thus we have

Theorem 3. Let $\sigma$ be a root of equation (4).

- If $\mathcal{P}^{\prime}(\sigma ; \delta, \nu, \kappa)<0$, the solution $\rho_{*}(\tau), \psi_{*}(\tau)$ with asymptotics (3) is unstable.

- If $\mathcal{P}^{\prime}(\sigma ; \delta, \nu, \kappa)=0$ and $\mp \mathcal{P}^{\prime \prime}(\sigma ; \delta, \nu, \kappa)>0$, the solution $\rho_{*}(\tau), \psi_{*}(\tau)$ with asymptotics (3), $\psi_{1}= \pm \phi$ is unstable.

- If $\mathcal{P}^{\prime}(\sigma ; \delta, \nu, \kappa)=0, \mathcal{P}^{\prime \prime}(\sigma ; \delta, \nu, \kappa)=0$, and $\mathcal{P}^{\prime \prime \prime}(\sigma ; \delta, \nu, \kappa)<0$, the solution $\rho_{*}(\tau), \psi_{*}(\tau)$ with asymptotics (6) is unstable.

- If $\mathcal{P}^{\prime}(\sigma ; \delta, \nu, \kappa)=0, \mathcal{P}^{\prime \prime}(\sigma ; \delta, \nu, \kappa)=0$, and $\mathcal{P}^{\prime \prime \prime}(\sigma ; \delta, \nu, \kappa)=0$, the solution $\rho_{*}(\tau), \psi_{*}(\tau)$ with asymptotics (7), $\psi_{1}=-\xi$ is unstable.

Let us consider the following cases that are not covered by Theorem 3 :

Case I: $\mathcal{P}^{\prime}(\sigma ; \delta, \nu, \kappa)>0$.

Case II: $\mathcal{P}^{\prime}(\sigma ; \delta, \nu, \kappa)=0, \pm \mathcal{P}^{\prime \prime}(\sigma ; \delta, \nu)>0$ and $\hat{\psi}(\tau)= \pm \phi \tau^{-\frac{1}{2}}+\mathcal{O}\left(\tau^{-1}\right)$ as $\tau \rightarrow \infty$.

Case III: $\mathcal{P}^{\prime}(\sigma ; \delta, \nu, \kappa)=0, \mathcal{P}^{\prime \prime}(\sigma ; \delta, \nu, \kappa)=0$, and $\mathcal{P}^{\prime \prime \prime}(\sigma ; \delta, \nu, \kappa)>0$.

Case IV: $\mathcal{P}^{\prime}(\sigma ; \delta, \nu, \kappa)=0, \mathcal{P}^{\prime \prime}(\sigma ; \delta, \nu, \kappa)=0, \mathcal{P}^{\prime \prime \prime}(\sigma ; \delta, \nu, \kappa)=0$ and $\hat{\psi}(\tau)=\xi \tau^{-\frac{1}{4}}+\mathcal{O}\left(\tau^{-\frac{1}{2}}\right)$ as $\tau \rightarrow \infty$. 
In these cases, the roots of the characteristic equation are complex. In particular,

$$
\begin{aligned}
& z_{ \pm}(\tau)= \pm i \tau^{\frac{1}{2}}(4 \lambda)^{\frac{1}{4}} \sqrt{\mathcal{P}^{\prime}(\sigma ; \delta, \nu, \kappa)}+\mathcal{O}(1), \quad \text { in Case I } \\
& z_{ \pm}(\tau)= \pm i \tau^{\frac{1}{4}}(4 \lambda)^{\frac{1}{4}} \sqrt{\left|\phi \mathcal{P}^{\prime \prime}(\sigma ; \delta, \nu, \kappa)\right|}+\mathcal{O}(1), \quad \text { in Case II, } \\
& z_{ \pm}(\tau)= \pm i \tau^{\frac{1}{6}} \lambda^{\frac{1}{4}} \sqrt{\chi^{2} \mathcal{P}^{\prime \prime \prime}(\sigma ; \delta, \nu, \kappa)}+\mathcal{O}(1), \quad \text { in Case III, } \\
& z_{ \pm}(\tau)= \pm 2 i \tau^{\frac{1}{8}} \lambda^{\frac{1}{4}} \xi^{\frac{3}{2}}+\mathcal{O}(1), \quad \text { in Case IV, }
\end{aligned}
$$

and $\Re z_{ \pm}(\tau)=\mathcal{O}(1)$ as $\tau \rightarrow \infty$. In such cases, the linear stability analysis fails (see, for example, 32, 33]), and the nonlinear terms of the equations must be taken into account.

3.2. Lyapunov functions. Let us specify the definition of stability that will be used in this section.

Definition 1. The solution $\rho_{*}(\tau), \psi_{*}(\tau)$ to system (1) is stable with the weights $\tau^{w_{1}}$ and $\tau^{w_{2}}$ as $\tau \geq \tau_{0}$ if for all $\epsilon>0$ there exists $\delta_{\epsilon}>0$ such that for all $\left(\rho^{0}, \psi^{0}\right):\left(\rho^{0}-\rho_{*}\left(\tau_{0}\right)\right)^{2}+\left(\psi^{0}-\psi_{*}\left(\tau_{0}\right)\right)^{2}<\delta_{\epsilon}^{2}$ the solution $\rho(\tau), \psi(\tau)$ to system (11) with initial data $\rho\left(\tau_{0}\right)=\rho^{0}, \psi\left(\tau_{0}\right)=\psi^{0}$ satisfies the following inequality:

$$
\left(\rho(\tau)-\rho_{*}(\tau)\right)^{2} \tau^{2 w_{1}}+\left(\psi(\tau)-\psi_{*}(\tau)\right)^{2} \tau^{2 w_{2}}<\epsilon^{2}
$$

for all $\tau>\tau_{0}$.

This definition modifies classical concept of stability with $w_{1}=w_{2}=0$. Inequality (9) can be considered as the estimate for the norm in the space of continuous functions with the weights $\tau^{w_{1}}$ and $\tau^{w_{2}}$. Thus, if the solution is stable with the weights $\tau^{w_{1}}, \tau^{w_{2}}$ and $w_{1}, w_{2} \geq 0$, then the perturbed solutions with initial data sufficiently close to $\rho_{*}(\tau), \psi_{*}(\tau)$ have the following asymptotics: $\rho(\tau)=$ $\rho_{*}(\tau)+\mathcal{O}\left(\tau^{-w_{1}}\right), \psi(\tau)=\psi_{*}(\tau)+\mathcal{O}\left(\tau^{-w_{2}}\right)$ as $\tau \rightarrow \infty$.

Theorem 4. Let $\sigma$ be a root of equation (4).

- In Case I, the solution $\rho_{*}(\tau), \psi_{*}(\tau)$ with asymptotics (3) is stable.

- In Case II, the solution $\rho_{*}(\tau), \psi_{*}(\tau)$ with asymptotics (3) is stable with the weights $\tau^{3 / 4}$ and $\tau^{1 / 2}$.

- In Case III, the solution $\rho_{*}(\tau), \psi_{*}(\tau)$ with asymptotics (6) is stable with the weights $\tau^{2 / 3}$ and $\tau^{1 / 3}$.

- In Case IV, the solution $\rho_{*}(\tau), \psi_{*}(\tau)$ with asymptotics (7) is stable with the weights $\tau^{5 / 8}$ and $\tau^{1 / 4}$.

Proof. Note that system (8) can be rewritten in a near-Hamiltonian form:

$$
\frac{d R}{d \tau}=-\partial_{\Psi} H(R, \Psi, \tau), \quad \frac{d \Psi}{d \tau}=\partial_{R} H(R, \Psi, \tau)+F(R, \Psi, \tau),
$$

where

$$
\begin{aligned}
H(R, \Psi, \tau):= & \rho_{*} R^{2}+\alpha\left(\cos \left(\psi_{*}+\Psi\right)-\cos \psi_{*}+\Psi \sin \psi_{*}\right) \\
& -\frac{\beta \rho_{*}}{2}\left(\cos \left(2 \psi_{*}+2 \Psi+\nu\right)-\cos \left(2 \psi_{*}+\nu\right)+2 \Psi \sin \left(2 \psi_{*}+\nu\right)\right) \\
& +\gamma R \Psi+\frac{R^{3}}{3}-\frac{\beta R}{2}\left(\cos \left(2 \psi_{*}+2 \Psi+\nu\right)-\cos \left(2 \psi_{*}+\nu\right)\right)
\end{aligned}
$$

and

$$
F(R, \Psi, \tau):=\alpha\left(\frac{\cos \left(\psi_{*}+\Psi\right)}{\rho_{*}+R}-\frac{\cos \psi_{*}}{\rho_{*}}\right)-\frac{\beta}{2}\left(\cos \left(2 \psi_{*}+2 \Psi+\nu\right)-\cos \left(2 \psi_{*}+\nu\right)\right)-\gamma \Psi
$$


Taking into account the asymptotic formulas for the particular solutions $\rho_{*}(\tau)=\sqrt{\lambda \tau}(1+\hat{\rho}(\tau))$ and $\psi_{*}(\tau)=\sigma+\hat{\psi}(\tau)$, we obtain the following asymptotic estimates:

$$
\begin{aligned}
H= & \tau^{\frac{1}{2}}\left(\sqrt{\lambda} R^{2}+\int_{0}^{\Psi} \mathcal{P}(\sigma+\zeta ; \delta, \nu, \kappa) d \zeta\right)+\tau^{\frac{1}{2}} \hat{\psi}\left(\mathcal{P}(\sigma+\Psi ; \delta, \nu, \kappa)-\mathcal{P}^{\prime}(\sigma ; \delta, \nu, \kappa) \Psi\right) \\
& +\tau^{\frac{1}{2}} \frac{\hat{\psi}^{2}}{2}\left(\mathcal{P}^{\prime}(\sigma+\Psi ; \delta, \nu, \kappa)-\mathcal{P}^{\prime \prime}(\sigma ; \delta, \nu, \kappa) \Psi-\mathcal{P}^{\prime}(\sigma ; \delta, \nu, \kappa)\right) \\
& +\tau^{\frac{1}{2}} \frac{\hat{\psi}^{3}}{6}\left(\mathcal{P}^{\prime \prime}(\sigma+\Psi ; \delta, \nu, \kappa)-\mathcal{P}^{\prime \prime \prime}(\sigma ; \delta, \nu, \kappa) \Psi-\mathcal{P}^{\prime \prime}(\sigma ; \delta, \nu, \kappa)\right) \\
& +\gamma_{0} R \Psi+\frac{R^{3}}{3}-\frac{\beta_{0} R}{2}(\cos (2 \sigma+2 \Psi+\nu)-\cos (2 \sigma+\nu))+ \\
& +\beta_{0} R \hat{\psi}(\sin (2 \sigma+2 \Psi+\nu)-\sin (2 \sigma+\nu))+\mathcal{O}\left(\hat{\psi}^{2}\right)+\mathcal{O}\left(\tau^{\frac{1}{2}} \hat{\psi}^{4}\right)+\mathcal{O}\left(\tau^{-\frac{1}{2}}\right)
\end{aligned}
$$

and

$$
F=\frac{1}{\sqrt{\lambda}} \int_{0}^{\Psi}(\mathcal{P}(\sigma+\zeta ; \delta, \nu, \kappa)-2 \kappa) d \zeta+\mathcal{O}(\hat{\psi})+\mathcal{O}\left(\tau^{-\frac{1}{2}}\right)
$$

as $\tau \rightarrow \infty$, for all $(R, \Psi) \in B\left(d_{*}\right)$, where

$$
B\left(d_{*}\right):=\left\{(R, \Psi) \in \mathbb{R}^{2}: d=\sqrt{R^{2}+\Psi^{2}} \leq d_{*}\right\}, \quad d_{*}=\text { const }>0 .
$$

Consider Case I, when $\sigma$ is the simple root to equation (44). In this case, $\hat{\psi}=\theta \tau^{-1}+\mathcal{O}\left(\tau^{-3 / 2}\right)$, and we get

$$
H=\tau^{\frac{1}{2}}\left(\sqrt{\lambda} R^{2}+\omega_{1}^{2} \frac{\Psi^{2}}{2}+\mathcal{O}\left(d^{3}\right)\right)+\mathcal{O}\left(\tau^{-\frac{1}{2}} d^{2}\right), \quad F=-2 \gamma_{0} \Psi+\mathcal{O}\left(d^{2}\right)+\mathcal{O}\left(\tau^{-\frac{1}{2}} d\right)
$$

as $d \rightarrow 0$ and $\tau \rightarrow \infty$, where $\omega_{1}^{2}=\mathcal{P}^{\prime}(\sigma ; \delta, \nu, \kappa)>0$. Note that the asymptotic estimates are uniform with respect to $(R, \Psi, \tau)$ in the domain $\left\{(R, \Psi, \tau) \in \mathbb{R}^{3}: d \leq d_{*}, \tau \geq \tau_{*}\right\}$, where $d_{*}, \tau_{*}=$ const $>0$. Consider the combination

$$
V_{1}(R, \Psi, \tau):=\tau^{-\frac{1}{2}}\left(H(R, \Psi, \tau)-\gamma_{0} R \Psi\right)
$$

as a Lyapunov function candidate for system (10). The function $V_{1}(R, \Psi, \tau)$ has the following asymptotics:

$$
V_{1}=\sqrt{\lambda} R^{2}+\omega_{1}^{2} \frac{\Psi^{2}}{2}+\mathcal{O}\left(d^{3}\right)+\mathcal{O}\left(\tau^{-\frac{1}{2}} d^{2}\right)
$$

as $d \rightarrow 0$ and $\tau \rightarrow \infty$. It can easily be checked that for all $0<\varkappa<1$ there exist $d_{1}>0$ and $\tau_{11}>0$ such that

$$
(1-\varkappa) W_{1}(R, \Psi) \leq V_{1}(R, \Psi, \tau) \leq(1+\varkappa) W_{1}(R, \Psi)
$$

for all $(R, \Psi, \tau) \in D_{W_{1}}\left(d_{1}, \tau_{11}\right)$, where

$$
D_{W_{1}}\left(d_{1}, \tau_{11}\right):=\left\{(R, \Psi, \tau) \in \mathbb{R}^{3}: W_{1}(R, \Psi) \leq d_{1}^{2}, \tau \geq \tau_{11}\right\}, \quad W_{1}(R, \Psi):=\sqrt{\lambda} R^{2}+\omega_{1}^{2} \frac{\Psi^{2}}{2} .
$$

The total derivative of the function $V_{1}(R, \Psi, \tau)$ with respect to $\tau$ along the trajectories of system (10) has the form:

$$
\left.\frac{d V_{1}}{d \tau}\right|_{(10)}=\frac{\partial V_{1}}{\partial \tau}-\frac{\partial V_{1}}{\partial R} \partial_{\Psi} H+\frac{\partial V_{1}}{\partial \Psi}\left(\partial_{R} H+F\right)=-2 \gamma_{0}\left(\sqrt{\lambda} R^{2}+\omega_{1}^{2} \frac{\Psi^{2}}{2}+\mathcal{O}\left(d^{3}\right)\right)+\mathcal{O}\left(\tau^{-\frac{1}{2}} d^{2}\right)
$$

as $d \rightarrow 0$ and $\tau \rightarrow \infty$. Hence, for all $0<\varkappa<1$ there exist $d_{2}>0$ and $\tau_{12}>0$ such that

$$
\left.\frac{d V_{1}}{d \tau}\right|_{(10)} \leq-2 \gamma_{\varkappa} V_{1}, \quad \gamma_{\varkappa}:=\gamma_{0}\left(\frac{1-\varkappa}{1+\varkappa}\right)>0 \text {, }
$$


for all $(R, \Psi, \tau) \in D_{W_{1}}\left(d_{2}, \tau_{12}\right)$. Thus, for all $0<\varepsilon<d_{0}$ there exist $\delta_{\varepsilon}:=\varepsilon \sqrt{(1-\varkappa) /(1+\varkappa)} / 2$ such that

$$
\sup _{W_{1} \leq \delta_{\varepsilon}^{2}} V_{1}(R, \Psi, \tau) \leq(1+\varkappa) \delta_{\varepsilon}^{2}<(1-\varkappa) \varepsilon^{2} \leq \inf _{W_{1}=\varepsilon^{2}} V_{1}(R, \Psi, \tau)
$$

for all $\tau>\tau_{0}$, where $d_{0}=\min \left\{d_{1}, d_{2}\right\}$ and $\tau_{0}=\max \left\{\tau_{11}, \tau_{12}\right\}$. The last estimates and the negativity of the total derivative of the function $V_{1}(R, \Psi, \tau)$ ensure that any solution of system (10) with initial data $W_{1}\left(R\left(\tau_{0}\right), \Psi\left(\tau_{0}\right)\right) \leq \delta_{\varepsilon}^{2}$ cannot leave the domain $\left\{(R, \Psi) \in \mathbb{R}^{2}: W_{1}(R, \Psi) \leq \varepsilon^{2}\right\}$ as $\tau>\tau_{0}$. Hence, the fixed point $(0,0)$ is stable as $\tau>\tau_{0}$. The stability on the finite time interval $\left(0, \tau_{0}\right]$ follows from the theorem on the continuity of the solutions to the Cauchy problem with respect to the initial data.

Consider Case II. Let $\sigma$ be a root of multiplicity 2 to equation (4) such that $\mathcal{P}^{\prime}(\sigma ; \delta, \nu, \kappa)=0$, $\pm \mathcal{P}^{\prime \prime}(\sigma ; \delta, \nu, \kappa) \hat{\psi}=\omega_{2}^{2} \tau^{-1 / 2}+\mathcal{O}\left(\tau^{-1}\right)$, where $\omega_{2}^{2}=\phi \mathcal{P}^{\prime \prime}(\sigma ; \delta, \nu, \kappa)>0$. Hence,

$$
H=\tau^{\frac{1}{2}}\left(\sqrt{\lambda} R^{2}+\mathcal{P}^{\prime \prime}(\sigma ; \delta, \nu, \kappa) \frac{\Psi^{3}}{6}+\mathcal{O}\left(d^{4}\right)\right)+\omega_{2}^{2} \frac{\Psi^{2}}{2}+\mathcal{O}\left(d^{3}\right)+\mathcal{O}\left(\tau^{-\frac{1}{2}} d^{2}\right)
$$

as $d \rightarrow 0$ and $\tau \rightarrow \infty$. In this case, $H(R, \Psi, \tau)$ is sign indefinite and the function $V_{1}(R, \Psi, \tau)$ can not be used as a Lyapunov function. Consider the change of variables

$$
R(\tau)=\tau^{-\frac{3}{4}} r(\tau), \quad \Psi(\tau)=\tau^{-\frac{1}{2}} \varphi(\tau)
$$

in system (10). The transformed system is

$$
\frac{d r}{d \tau}=-\partial_{\varphi} H_{2}(r, \varphi, \tau), \quad \frac{d \varphi}{d \tau}=\partial_{r} H_{2}(r, \varphi, \tau)+F_{2}(r, \varphi, \tau)
$$

where

$$
H_{2}(r, \varphi, \tau):=\tau^{\frac{5}{4}} H\left(\tau^{-\frac{3}{4}} r, \tau^{-\frac{1}{2}} \varphi, \tau\right)-\tau^{-1} \frac{3 r \varphi}{4}, \quad F_{2}(r, \varphi, \tau):=\tau^{\frac{1}{2}} F\left(\tau^{-\frac{3}{4}} r, \tau^{-\frac{1}{2}} \varphi, \tau\right)+\tau^{-1} \frac{5 \varphi}{4} .
$$

Taking into account (3), we see that

$$
H_{2}=\tau^{\frac{1}{4}}\left(\sqrt{\lambda} R^{2}+\omega_{2}^{2} \frac{\varphi^{2}}{2}+\mathcal{P}^{\prime \prime}(\sigma ; \delta, \nu, \kappa) \frac{\varphi^{3}}{6}\right)+\mathcal{O}\left(\Delta^{2}\right), \quad F_{2}=-2 \gamma_{0} \varphi+\mathcal{O}\left(\Delta \tau^{-\frac{1}{2}}\right)
$$

as $t \rightarrow \infty$ and $\Delta=\sqrt{r^{2}+\varphi^{2}} \rightarrow 0$. Note that the function $H_{2}(r, \varphi, \tau)$ is suitable for the basis of a Lyapunov function candidate:

$$
V_{2}(r, \varphi, \tau)=\tau^{-\frac{1}{4}}\left(H_{2}(r, \varphi, \tau)-\gamma_{0} r \varphi\right)
$$

It follows easily that for all $0<\varkappa<1$ there exist $\Delta_{1}>0$ and $\tau_{1}>0$ such that

$$
(1-\varkappa) W_{2}(r, \varphi) \leq V_{2}(r, \varphi, \tau) \leq(1+\varkappa) W_{2}(r, \varphi)
$$

for all $(r, \varphi, \tau) \in D_{W_{2}}\left(\Delta_{1}, \tau_{1}\right)$, where $W_{2}(r, \varphi):=\sqrt{\lambda} r^{2}+\omega_{2}^{2} \varphi^{2} / 2$. The total derivative of the function $V_{2}(r, \varphi, \tau)$ has a sign definite leading term of the asymptotics:

$$
\begin{aligned}
\left.\frac{d V_{2}}{d \tau}\right|_{(13)} & =\frac{\partial V_{2}}{\partial \tau}+\frac{\partial V_{2}}{\partial \varphi} F_{2}+\gamma_{0} \tau^{-\frac{1}{4}}\left(\varphi \frac{\partial H_{2}}{\partial \varphi}-r \frac{\partial H_{2}}{\partial r}\right) \\
& =-2 \gamma_{0}\left(\sqrt{\lambda} r^{2}+\omega_{2}^{2} \frac{\varphi^{2}}{2}+\mathcal{O}\left(\Delta^{3}\right)\right)+\mathcal{O}\left(\tau^{-\frac{1}{4}} \Delta^{2}\right)
\end{aligned}
$$

as $\Delta \rightarrow 0$ and $\tau \rightarrow \infty$. Hence, for all $0<\varkappa<1$ there exist $\Delta_{2}>0$ and $\tau_{2}>0$ such that

$$
\left.\frac{d V_{2}}{d \tau}\right|_{(13)} \leq-2 \gamma_{\varkappa} V_{2} \leq 0
$$

for all $(r, \varphi, \tau) \in D_{W_{2}}\left(\Delta_{2}, \tau_{2}\right)$. As above, the last inequality implies the stability of the equilibrium $(0,0)$ to system (13). Returning to the original variables, we obtain the result of the Theorem.

In Case III, $\sigma$ is a root of multiplicity 3 to equation (4) such that $\mathcal{P}^{\prime}(\sigma ; \delta, \nu, \kappa)=\mathcal{P}^{\prime \prime}(\sigma ; \delta, \nu, \kappa)=0$ and $\mathcal{P}^{\prime \prime \prime}(\sigma ; \delta, \nu, \kappa)>0$. The solution $\rho_{*}(\tau), \psi_{*}(\tau)$ has the asymptotics (6) , and $\hat{\psi}=\chi \tau^{-1 / 3}+\mathcal{O}\left(\tau^{-2 / 3}\right)$. In this case,

$$
H(R, \Psi, \tau)=\tau^{\frac{1}{2}}\left(\sqrt{\lambda} R^{2}+\mathcal{P}^{\prime \prime \prime}(\sigma ; \delta, \nu, \kappa) \frac{\Psi^{4}}{24}+\mathcal{O}\left(d^{5}\right)\right)+\mathcal{O}\left(\tau^{\frac{1}{6}} d^{3}\right)+\mathcal{O}\left(d^{2}\right)
$$


as $d \rightarrow 0$ and $\tau \rightarrow \infty$. Note that this function is sign indefinite in a neighborhood of the equilibrium and can not be used in the construction of a Lyapunov function. Indeed, if $\Psi \sim \epsilon^{1 / 4}$ and $\tau \sim \epsilon^{-1}$ as $\epsilon \rightarrow 0$, the leading and the remainder terms in the last expression can be of the same order. Consider the change of variables:

$$
R(\tau)=\tau^{-\frac{2}{3}} r(\tau), \quad \Psi(\tau)=\tau^{-\frac{1}{3}} \varphi(\tau)
$$

in system (10). It can easily be checked that the transformed system has the form

$$
\frac{d r}{d \tau}=-\partial_{\varphi} H_{3}(r, \varphi, \tau), \quad \frac{d \varphi}{d \tau}=\partial_{r} H_{3}(r, \varphi, \tau)+F_{3}(r, \varphi, \tau),
$$

where

$$
H_{3}(r, \varphi, \tau):=\tau H\left(\tau^{-\frac{2}{3}} r, \tau^{-\frac{1}{3}} \varphi, \tau\right)-\tau^{-1} \frac{2 r \varphi}{3}, \quad F_{3}(r, \varphi, \tau):=\tau^{\frac{1}{3}} F\left(\tau^{-\frac{2}{3}} r, \tau^{-\frac{1}{3}} \varphi, \tau\right)+\tau^{-1} \varphi .
$$

Using asymptotic formulas for the particular solution, we obtain

$$
H_{3}=\tau^{\frac{1}{6}}\left(\sqrt{\lambda} r^{2}+\omega_{3}^{2} \frac{\varphi^{2}}{2}-\mathcal{P}^{\prime \prime \prime}(\sigma ; \delta, \nu, \kappa) \frac{\varphi^{3}}{6}\left(\chi-\frac{\varphi}{4}\right)\right)+\mathcal{O}\left(\Delta^{2}\right), \quad F_{3}=-2 \gamma_{0} \varphi+\mathcal{O}\left(\Delta \tau^{-\frac{1}{3}}\right)
$$

as $\Delta \rightarrow 0$ and $\tau \rightarrow \infty$, where $\omega_{3}^{2}:=\chi^{2} \mathcal{P}^{\prime \prime \prime}(\sigma ; \delta, \nu) / 2>0$. Consider the combination

$$
V_{3}(r, \varphi, \tau)=\tau^{-\frac{1}{6}}\left(H_{3}(r, \varphi, \tau)-\gamma_{0} r \varphi\right)
$$

as a Lyapunov function candidate for system (15). It follows easily that for all $0<\varkappa<1$ there exist $\Delta_{3}>0$ and $\tau_{3}>0$ such that

$$
(1-\varkappa) W_{3}(r, \varphi) \leq V_{3}(r, \varphi, \tau) \leq(1+\varkappa) W_{3}(r, \varphi)
$$

for all $(r, \varphi, \tau) \in D_{W_{3}}\left(\Delta_{3}, \tau_{3}\right)$, where $W_{3}(r, \varphi):=\sqrt{\lambda} r^{2}+\omega_{3}^{2} \varphi^{2} / 2$. The derivative of this function with respect to $\tau$ along the trajectories of system (15) satisfies:

$$
\left.\frac{d V_{3}}{d \tau}\right|_{\text {(15) }}=-2 \gamma_{0}\left(\sqrt{\lambda} r^{2}+\omega_{3}^{2} \frac{\varphi^{2}}{2}+\mathcal{O}\left(\Delta^{3}\right)\right)+\mathcal{O}\left(\tau^{-\frac{1}{6}} \Delta^{2}\right)
$$

as $\Delta \rightarrow 0$ and $\tau \rightarrow \infty$. Hence, for all $0<\varkappa<1$ there exist $\Delta_{4}>0$ and $\tau_{4}>0$ such that

$$
\left.\frac{d V_{3}}{d \tau}\right|_{[15)} \leq-2 \gamma_{\varkappa} V_{3} \leq 0
$$

for all $(r, \varphi, \tau) \in D_{W_{3}}\left(\Delta_{4}, \tau_{4}\right)$. The last inequality implies that the fixed point $(0,0)$ of system (15) is stable and the solution $\rho_{*}(\tau), \psi_{*}(\tau)$ is stable with the weights $\tau^{2 / 3}$ and $\tau^{1 / 3}$.

Finally, consider Case IV. The change of variables

$$
R(\tau)=\tau^{-\frac{5}{8}} r(\tau), \quad \Psi(\tau)=\tau^{-\frac{1}{4}} \varphi(\tau)
$$

transforms system (10) into

$$
\frac{d r}{d \tau}=-\partial_{\varphi} H_{4}(r, \varphi, \tau), \quad \frac{d \varphi}{d \tau}=\partial_{r} H_{4}(r, \varphi, \tau)+F_{4}(r, \varphi, \tau),
$$

where

$$
H_{4}(r, \varphi, \tau):=\tau^{\frac{7}{8}} H\left(\tau^{-\frac{5}{8}} r, \tau^{-\frac{1}{4}} \varphi, \tau\right)-\tau^{-1} \frac{5 r \varphi}{8}, \quad F_{4}(r, \varphi, \tau):=\tau^{-1} \frac{7 \varphi}{8}+\tau^{\frac{1}{4}} F\left(\tau^{-\frac{5}{8}} r, \tau^{-\frac{1}{4}} \varphi, \tau\right) .
$$

Taking into account (7), it can easily be checked that

$$
H_{4}(r, \varphi, \tau)=\tau^{\frac{1}{8}}\left(\sqrt{\lambda} R^{2}+\omega_{4}^{2} \frac{\varphi^{2}}{2}+\frac{\xi^{2} \varphi^{3}}{12}+\frac{\xi \varphi^{4}}{8}+\frac{\varphi^{5}}{40}\right)+\mathcal{O}\left(\Delta^{2}\right), \quad F_{4}(r, \varphi, \tau)=-2 \gamma_{0} \varphi+\mathcal{O}\left(\Delta \tau^{-\frac{1}{8}}\right)
$$

as $\Delta \rightarrow 0$ and $\tau \rightarrow \infty$, where $\omega_{4}^{2}:=\xi^{3} / 4>0$. Consider

$$
V_{4}(r, \varphi, \tau)=\tau^{-\frac{1}{8}}\left(H_{4}(r, \varphi, \tau)-\gamma_{0} r \varphi\right)
$$

as a Lyapunov function candidate to system (17). We see that for all $0<\varkappa<1$ there exist $\Delta_{5}>0$ and $\tau_{5}>0$ such that

$$
(1-\varkappa) W_{4}(r, \varphi) \leq V_{4}(r, \varphi, \tau) \leq(1+\varkappa) W_{4}(r, \varphi)
$$


for all $(r, \varphi, \tau) \in D_{W_{4}}\left(\Delta_{5}, \tau_{5}\right)$, where $W_{4}(r, \varphi):=\sqrt{\lambda} r^{2}+\omega_{4}^{2} \varphi^{2} / 2$. The total derivative of $V_{4}$ with respect to $\tau$ satisfies:

$$
\left.\frac{d V_{4}}{d \tau}\right|_{\text {(17] }}=-2 \gamma_{0}\left(\sqrt{\lambda} r^{2}+\omega_{4}^{2} \frac{\varphi^{2}}{2}+\mathcal{O}\left(\Delta^{3}\right)\right)+\mathcal{O}\left(\tau^{-\frac{1}{8}} \Delta^{2}\right)
$$

as $\Delta \rightarrow 0$ and $\tau \rightarrow \infty$. Hence, for all $0<\varkappa<1$ there exist $\Delta_{6}>0$ and $\tau_{6}>0$ such that

$$
\left.\frac{d V_{4}}{d \tau}\right|_{(17)} \leq-2 \gamma_{\varkappa} V_{4} \leq 0
$$

for all $(r, \varphi, \tau) \in D_{W_{4}}\left(\Delta_{6}, \tau_{6}\right)$. The last inequality implies that the fixed point $(0,0)$ of system (15) is stable. Returning to the variables $(\rho, \psi)$ we obtain the result of the theorem.

Thus, the particular autoresonant solutions $\rho_{*}(\tau), \psi_{*}(\tau)$ with power-law asymptotics are stable in all four cases. The stability ensures the existence of a family of solutions with a similar behaviour. In particular, we have the following.

Corollary 1. There exist $\Delta_{*}>0$ and $T_{*}>0$ such that for all $\left(\rho^{0}, \psi^{0}\right):\left(\rho^{0}-\rho_{*}\left(T_{*}\right)\right)^{2}+\left(\psi^{0}-\psi_{*}\left(T_{*}\right)\right)^{2}<$ $\Delta_{*}^{2}$ the solution $\rho(\tau), \psi(\tau)$ to system (11) with initial data $\rho\left(T_{*}\right)=\rho^{0}, \psi\left(T_{*}\right)=\psi^{0}$ has the following estimates as $\tau \rightarrow \infty$ :

$$
\begin{array}{lll}
\rho=\sqrt{\lambda \tau}+\mathcal{O}\left(\tau^{-\frac{1}{2}}\right), & \psi=\sigma+\mathcal{O}\left(\tau^{-1}\right) & \text { in Case I; } \\
\rho=\sqrt{\lambda \tau}+\mathcal{O}\left(\tau^{-\frac{1}{2}}\right), & \psi=\sigma+\mathcal{O}\left(\tau^{-\frac{1}{2}}\right) & \text { in Case II; } \\
\rho=\sqrt{\lambda \tau}+\mathcal{O}\left(\tau^{-\frac{1}{2}}\right), & \psi=\sigma+\mathcal{O}\left(\tau^{-\frac{1}{3}}\right) & \text { in Case III; } \\
\rho=\sqrt{\lambda \tau}+\mathcal{O}\left(\tau^{-\frac{1}{2}}\right), & \psi=\frac{\pi}{2}+\mathcal{O}\left(\tau^{-\frac{1}{4}}\right) & \text { in Case IV. }
\end{array}
$$

Proof. Consider Case I. Let $R(\tau), \Psi(\tau)$ be a solution to system (10) starting from the ball $B\left(\Delta_{*}\right)$ at $\tau=T_{*}$, where $\Delta_{*}=d_{0}$ and $T_{*}=\tau_{0}$ (see Theorem 44). Then it follows from (11) that the function $v(\tau)=V_{1}(R(\tau), \Psi(\tau), \tau)$ satisfies the inequality:

$$
\frac{d v}{d \tau} \leq-2 \gamma_{\varkappa} v
$$

as $\tau \geq T_{*}$, where $\gamma_{\varkappa}=\gamma_{0}(1-\varkappa) /(1+\varkappa), \varkappa \in(0,1)$. Integrating the last expression with respect to $\tau$, we obtain $0 \leq v(\tau) \leq v\left(T_{*}\right) \exp \left(-2 \gamma_{\varkappa}\left(\tau-T_{*}\right)\right)$, where $0 \leq v\left(T_{*}\right) \leq C_{*} \Delta_{*}^{2}, C_{*}=$ const. Thus we have $R(\tau)=\mathcal{O}\left(e^{-\gamma_{\varkappa} \tau}\right), \Psi(\tau)=\mathcal{O}\left(e^{-\gamma_{\varkappa} \tau}\right)$ as $\tau \rightarrow \infty$. Since $\rho(\tau)=\rho_{*}(\tau)+R(\tau), \psi(\tau)=\psi_{*}(\tau)+\Psi(\tau)$ and $\rho_{*}(\tau)=\sqrt{\lambda \tau}+\mathcal{O}\left(\tau^{-1 / 2}\right), \psi_{*}(\tau)=\sigma+\mathcal{O}\left(\tau^{-1}\right)$ as $\tau \rightarrow \infty$, we have (18).

Case II. Let $r(\tau), \varphi(\tau)$ be a solution to system (13) starting from $\left\{\left(r_{0}, \varphi_{0}\right): W_{2}\left(r_{0}, \varphi_{0}\right) \leq \Delta_{*}^{2}\right\}$ at $\tau=T_{*}$, where $\Delta_{*}=\min \left\{\Delta_{1}, \Delta_{2}\right\}, T_{*}=\max \left\{\tau_{1}, \tau_{2}\right\}$. From (14) it follows that the derivative of the function $v(\tau)=V_{2}(r(\tau), \varphi(\tau), \tau)$ satisfies (22) as $\tau \geq T_{*}$. By integrating this estimate with respect to $\tau$ and taking into account (12), we get $R(\tau)=\mathcal{O}\left(\tau^{-3 / 4} e^{-\gamma_{\varkappa} \tau}\right), \Psi(\tau)=\mathcal{O}\left(\tau^{-1 / 2} e^{-\gamma_{\varkappa} \tau}\right)$ as $\tau \rightarrow \infty$. Returning to the variables $(\rho, \psi)$ and using (3), we derive (19).

Case III. Let $r(\tau), \varphi(\tau)$ be a solution to system (15) with initial data from $\left\{\left(r_{0}, \varphi_{0}\right): W_{3}\left(r_{0}, \varphi_{0}\right) \leq\right.$ $\left.\Delta_{*}^{2}\right\}$ at $\tau=T_{*}$, where $\Delta_{*}=\min \left\{\Delta_{3}, \Delta_{4}\right\}, T_{*}=\max \left\{\tau_{3}, \tau_{4}\right\}$. From (16) it follows that the function $v(\tau)=V_{3}(r(\tau), \varphi(\tau), \tau)$ satisfies (22) as $\tau \geq T_{*}$. Hence, $R(\tau)=\mathcal{O}\left(\tau^{-2 / 3} e^{-\gamma_{\varkappa} \tau}\right), \Psi(\tau)=$ $\mathcal{O}\left(\tau^{-1 / 3} e^{-\gamma_{\varkappa} \tau}\right)$ as $\tau \rightarrow \infty$. Combining this with (마), we get (20).

Finally, consider Case IV. Let $r(\tau), \varphi(\tau)$ be a solution to system (17) with initial data from $\left\{\left(r_{0}, \varphi_{0}\right): W_{4}\left(r_{0}, \varphi_{0}\right) \leq \Delta_{*}^{2}\right\}$ at $\tau=T_{*}$, where $\Delta_{*}=\min \left\{\Delta_{5}, \Delta_{6}\right\}, T_{*}=\max \left\{\tau_{5}, \tau_{6}\right\}$. It follows easily that the function $v(\tau)=V_{4}(r(\tau), \varphi(\tau), \tau)$ satisfies (22) as $\tau \geq T_{*}$. Hence, $R(\tau)=\mathcal{O}\left(\tau^{-5 / 8} e^{-\gamma_{\varkappa} \tau}\right)$, $\Psi(\tau)=\mathcal{O}\left(\tau^{-1 / 4} e^{-\gamma_{\varkappa} \tau}\right)$ as $\tau \rightarrow \infty$. Combining this with (7), we get (21).

\section{Conclusion}

Thus, we have described possible autoresonant modes in oscillating systems with a combined excitation and a weak dissipation. The presence of dissipation in the model leads to exponential stability of a part of autoresonant modes in comparison with a systems without dissipation, where only polynomial 


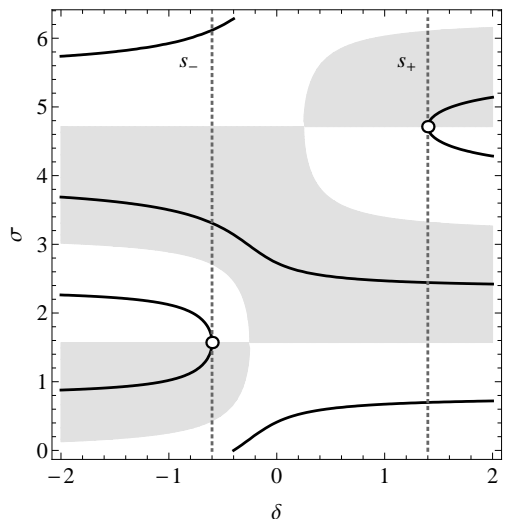

(a) $\kappa=0.4, \nu=\frac{\pi}{2}$

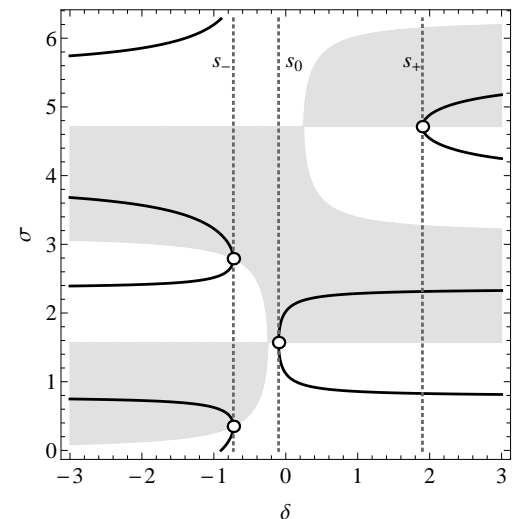

(b) $\kappa=0.9, \nu=\frac{\pi}{2}$

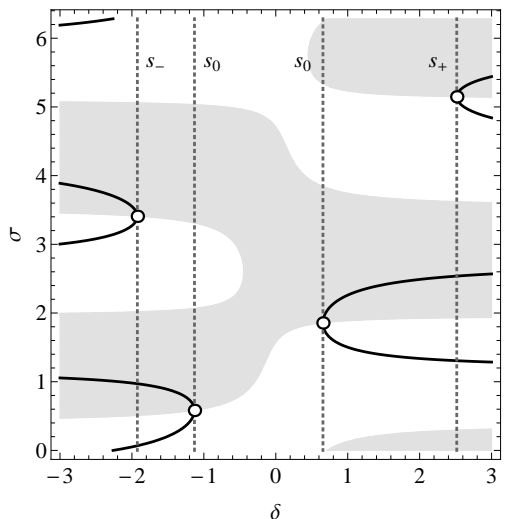

(c) $\kappa=1.6, \nu=\frac{\pi}{4}$

FiguRE 5. The roots to equation (4) as functions of the parameter $\delta$ (black solid lines). The vertical dotted lines correspond to $s_{-}, s_{0}$ and $s_{+}$. The shaded areas correspond to $\mathcal{P}^{\prime}(\sigma ; \delta, \nu, \kappa)>0$, where the particular solutions to system (11) with asymptotics (3) are stable.

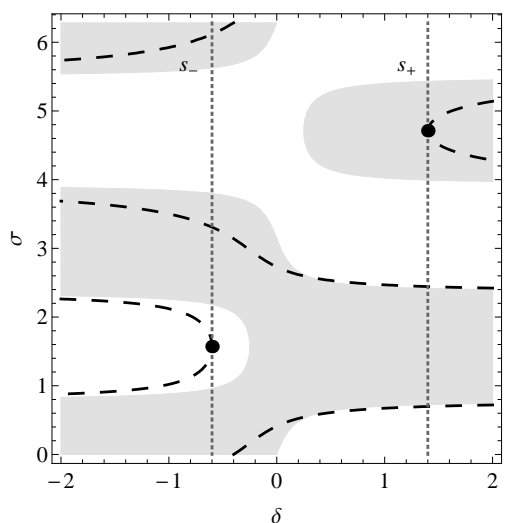

(a) $\kappa=0.4, \nu=\frac{\pi}{2}$

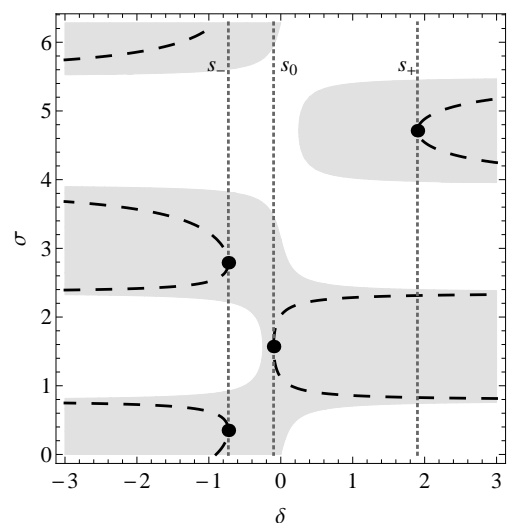

(b) $\kappa=0.9, \nu=\frac{\pi}{2}$

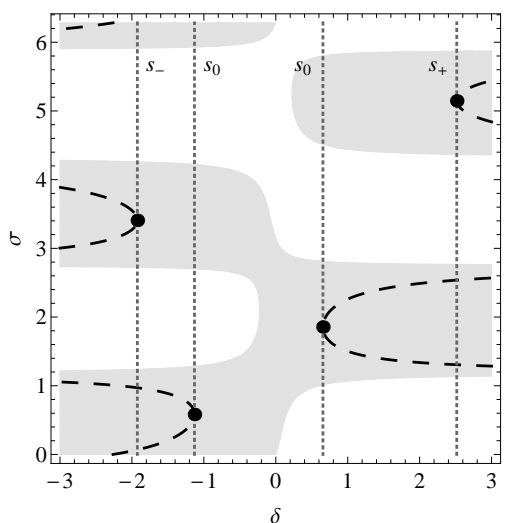

(c) $\kappa=1.6, \nu=\frac{\pi}{4}$

FiguRE 6. The roots to equation (44) as functions of the parameter $\delta$ (dashed lines). The vertical dotted lines correspond to $s_{-}, s_{0}$ and $s_{+}$. The black points correspond to the roots of multiplicity 2. The shaded areas correspond to $\mathcal{P}^{\prime \prime}(\sigma ; \delta, \nu, \kappa)>0$, where the particular solution to system (11) with asymptotics (3), $\psi_{0}=\sigma, \psi_{1}=\phi$ is stable.

stability takes place [27. Depending on the values of the excitation parameters and the dissipation coefficient, the system can have different number of autoresonant modes with different phase mismatch: $\psi(\tau) \sim \sigma$ as $\tau \rightarrow \infty$, where $\sigma$ is the root of equation (44). For every $\kappa=\gamma_{0} \sqrt{\lambda}>0$ the curves $s_{ \pm}$and $s_{0}$ consist of bifurcations points on the plane $(\delta, \nu)$, where $\delta=\beta_{0} \sqrt{\lambda}$. Outside of this curves the roots of equation (4) are simple and system (1) can have two or four different autoresonant modes. Their stability depends on the sign of the value $\mathcal{P}^{\prime}(\sigma ; \delta, \nu, \kappa)$ (see the shaded areas in Fig. (5)).

Some of the autoresonant modes coalesce, when the parameters $(\delta, \nu)$ pass through the bifurcation curves. The following cases are possible. (I) Equation (44) has three different roots: two simple roots and one root of multiplicity 2 (see Fig. 6, a). (II) Equation (4) has two different roots: one simple root and one root of multiplicity 3 (see Fig. 7, a), or two roots of multiplicity 2 (see Fig. 6, b). (III) Equation (4) has only one root: a root of multiplicity 2 (see Fig. 6, c), or a root of multiplicity 4 (see Fig. 7, b).

If $\sigma$ is the root of multiplicity 2, system (1) has two autoresonant modes associated with the particular solutions having asymptotics (3), where $\psi_{0}=\sigma$ and $\psi_{1}= \pm \phi$. In this case, the solution with $\psi_{1}= \pm \phi$ is stable if $\pm \mathcal{P}^{\prime \prime}(\sigma ; \delta, \nu, \kappa)>0$ (see Fig. 6). If $\sigma$ is the root of multiplicity 3, there 


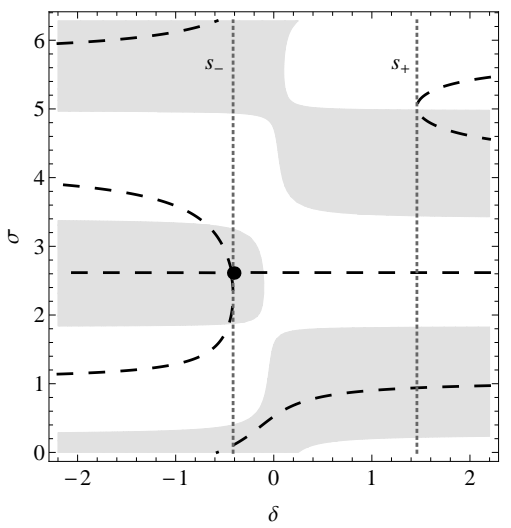

(a) $\kappa=0.5, \nu \approx 1.038$

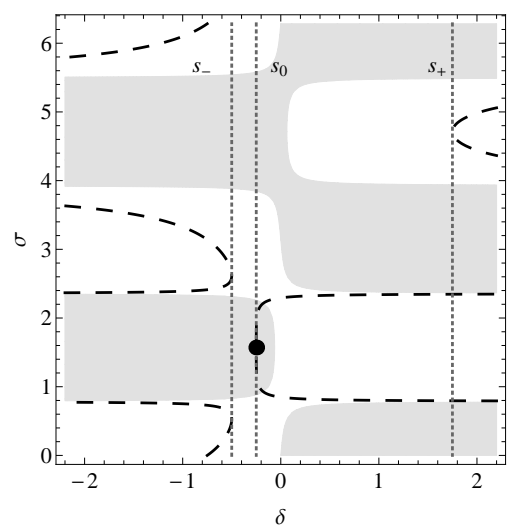

(b) $\kappa=0.75, \nu=\frac{\pi}{2}$

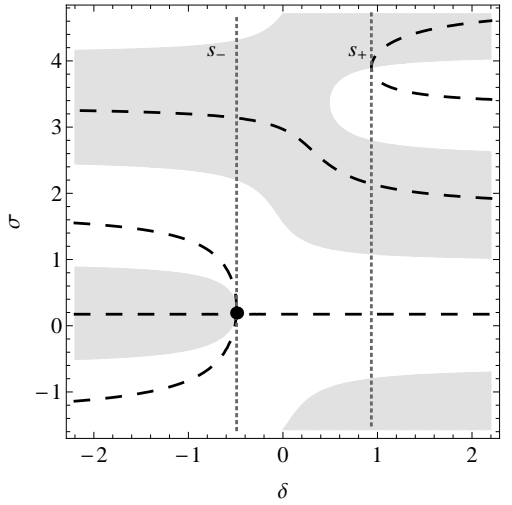

(c) $\kappa=\sqrt{0.3}, \nu \approx 2.79$

Figure 7. The roots to equation (44) as functions of the parameter $\delta$ (dashed lines). The vertical dotted lines correspond to $s_{-}, s_{0}$ and $s_{+}$. The black points correspond to the roots of multiplicity 3 and 4 . (a) The shaded areas correspond to $\mathcal{P}^{\prime \prime \prime}(\sigma ; \delta, \nu, \kappa)>0$, where the particular solution to system (11) with asymptotics (6) is stable. (b) The shaded areas correspond to $\mathcal{P}^{(4)}(\sigma ; \delta, \nu, \kappa)>0$. (c) The shaded areas correspond to $\mathcal{P}^{\prime}(\sigma ; \delta, \nu, \kappa)>0$
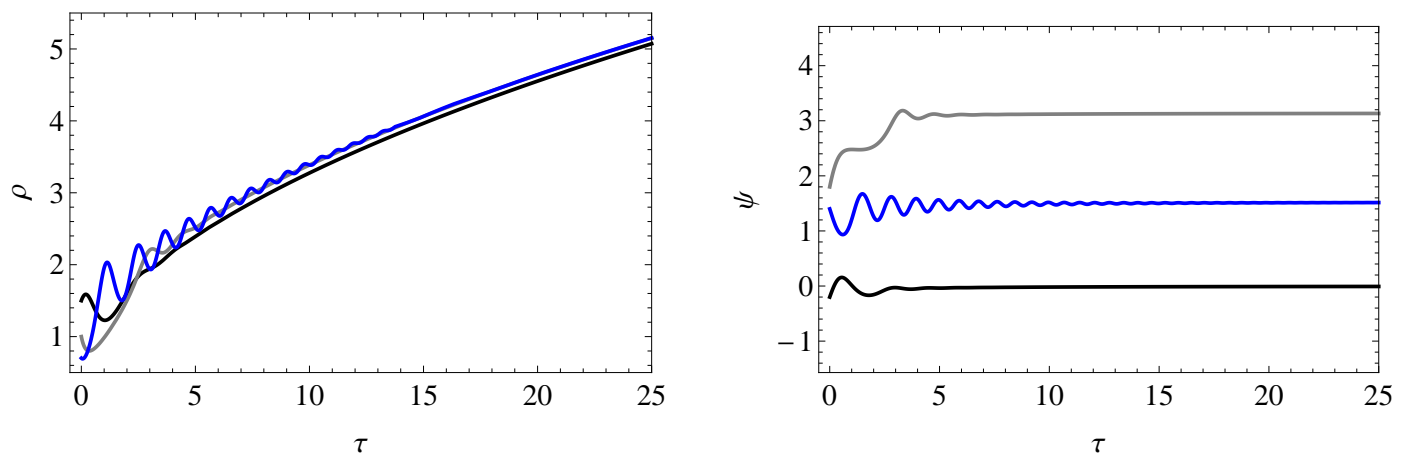

FiguRE 8. The evolution of $\rho(\tau)$ and $\psi(\tau)$ for solutions of system (10) with $\lambda=1$, $\alpha(\tau) \equiv \sqrt{\tau}, \beta(\tau) \equiv \delta, \gamma(\tau) \equiv \kappa$. The black curves correspond to $\delta=-2, \kappa=1$, $\nu=5 \pi / 6$. The gray curves correspond to $\delta=-2 / \sqrt{3}, \kappa=1, \nu=2 \pi / 3$. The blue curves correspond to $\delta=-3 / 2, \kappa=1 / 4, \nu=\pi / 6$.

is a mode corresponding to the particular solution with asymptotics (6), where $\psi_{0}=\sigma, \psi_{1}=0$ and $\psi_{2}=\chi$. This mode is stable if $\mathcal{P}^{\prime \prime \prime}(\sigma ; \delta, \nu, \kappa)>0$ (see Fig. 7) a). Finally, if $\sigma$ is the root of multiplicity 4 , there are two autoresonant modes with asymptotics (7), where $\psi_{0}=\sigma, \psi_{1}= \pm \xi$. In this case, the mode with $\psi_{1}=\xi$ is exponentially stable, while the mode with $\psi_{1}=-\xi$ is unstable (see Fig. 7, b).

Note that the combined excitation allows to expand the use of autoresonant method for control the dynamics of nonlinear systems. In particular, unstable autoresonant modes in systems with pure external excitation $(\delta=0)$ can be stabilized by switching on parametric pumping (see, for example, Fig. 7, c, where the mode with $\sigma=\arcsin (4 \kappa / 3)$ becomes stable as $\delta<-\sqrt{0.24})$. Moreover, for every $\gamma_{0}>0$, the parameters $\lambda>0, \beta_{0} \neq 0$ and $\nu \in[0, \pi)$ of the combined excitation can be chosen in such a way to guarantee the existence and stability of autoresonant mode with any prescribed phase shift $\psi(\tau) \approx \sigma, \sigma \in[0,2 \pi)$. For example, for $\sigma=0$, we should take $\nu=\pi-\arcsin \left(-\kappa \delta^{-1}\right)$ and $\delta<-\sqrt{\kappa^{2}+1 / 4}$. In this case, $\mathcal{P}(\sigma ; \delta, \nu, \kappa)=0, \mathcal{P}^{\prime}(\sigma ; \delta, \nu, \kappa)>0$ and Theorem 4 is applicable. Similarly, for $\sigma=\pi$, we should take $\nu=\pi-\arcsin \left(-\kappa \delta^{-1}\right)$ and $\delta<-\kappa$. For $\sigma=\pi / 2$, one can take $(\delta, \nu, \kappa)$ such that $0<(\kappa-1) \delta^{-1} \leq 1, \nu=\arcsin \left((\kappa-1) \delta^{-1}\right)$ and $\delta \cos \nu<0$ (see Fig. 8). 


\section{ACKNOWLEDGEMEnTS}

The research presented in Section 3 is funded in the framework of executing the development program of Scientific Educational Mathematical Center of Privolzhsky Federal Area, additional agreement no. 075-02-2020-1421/1 to agreement no. 075-02-2020-1421.

\section{REFERENCES}

[1] L. Friedland, "Autoresonance in nonlinear systems", Scholarpedia 4(1): 5473 (2009).

[2] V. I. Veksler, "A new method of acceleration of relativistic particles", J. Phys. USSR 9, 153-158 (1945).

[3] E. M. McMillan, "The synchrotron - a proposed high energy particle accelerator", Phys. Rev. 68, 143-144 (1945)

[4] R.J. Greenberg, "Evolution of satellite resonances by tidal dissipation", Astron. J. 78, 338-346 (1973).

[5] A.T. Sinclair, "On the origin of commensurabilities amongst satellites of Saturn", Mon. Not. Roy. Astron. Soc. 160, 169-187 (1978).

[6] J. Fajans, E. Gilson, and L. Friedland, "Second harmonic autoresonant control of the $\mathrm{l}=1$ diocotron mode in pure electron plasmas", Phys. Rev. E 62, 4131 (2000).

[7] M. Yu. Uleysky, E. V. Sosedko, and D. V. Makarov, "Autoresonant cooling of particles in spatially periodic potentials", Tech. Phys. Lett. 36, 1082-1084 (2010).

[8] G. B. Andresen et al. (ALPHA Collaboration). "Autoresonant excitation of antiproton plasmas", Phys. Rev. Lett. 106, 025002 (2011).

[9] G. Klughertz, P.-A. Hervieux, and G. Manfredi, "Autoresonant control of the magnetization switching in single-domain nanoparticles", J. Phys. D: Appl. Phys. 47, 34 (2014).

[10] L. Friedland, G. Marcus, J. S. Wurtele, and P. Michel "Excitation and control of large amplitude standing ion acoustic waves", Phys. Plasmas 26, 092109 (2019).

[11] A. G. Shagalov and L. Friedland, "Narrow autoresonant magnetization structures in finite-length ferromagnetic nanoparticle", Phys. Rev. E 100, 032208 (2019).

[12] S.V. Batalov, A.G. Shagalov, and L. Friedland, "Autoresonant excitation of Bose-Einstein condensates", Phys. Rev. E 97, 032210 (2018).

[13] L. Friedland and A.G. Shagalov, "Standing autoresonant plasma waves", J. Plasma Phys. 86, 825860301 (2020).

[14] L. Friedland, "Efficient capture of nonlinear oscillations into resonance", J. Phys. A: Math. Theor. 41, 415101 (2008).

[15] L. A. Kalyakin, "Asymptotic analysis of autoresonance models", Rus. Math. Surv. 63, 791-857 (2008).

[16] A.I. Neishtadt, A.A. Vasiliev, A.V. Artemyev, "Capture into resonance and escape from it in a forced nonlinear pendulum", Regul Chaot Dyn. 18, 686-696 (2013).

[17] J. Fajans, E. Gilson, and L. Friedland, "The effect of damping on autoresonant excitation", Phys. Plasmas 8, 423-427 (2001).

[18] L. A. Kalyakin, "Asymptotic behavior of solutions of equations of main resonance", Theoret. and Math. Phys. 137, 1476-1484 (2003).

[19] L. A. Kalyakin, M. A. Shamsutdinov, "Autoresonant asymptotics in an oscillating system with weak dissipation", Theor. Math. Phys. 160 960-967 (2009).

[20] S. Glebov, O. Kiselev, N. Tarkhanov, "Autoresonance in a dissipative system", J. Phys. A. 43, 215203 (2010).

[21] A. Kovaleva, "Autoresonance in weakly dissipative Klein-Gordon chains", Physica D. 402, 132284 (2020).

$[22]$ E. Khain and B. Meerson, "Parametric autoresonance", Phys. Rev. E 64, 036619 (2001).

[23] M. Asaf, B. Meerson, "Parametric autoresonance of Faraday waves", Phys. Rev. E 72, 016310 (2005).

[24] O.A. Sultanov, "Stability of capture into parametric autoresonance", Proc. Steklov Inst. Math. 295, suppl. $1,156-167$ (2016).

[25] O. M. Kiselev, "Asymptotic behaviour of measure for captured trajectories into parametric autoresonance", Nonlinear Dyn. 91, 1977-1983 (2018).

[26] O. Sultanov, "Capture into parametric autoresonance in the presence of noise", Comm. Nonlin. Sc. Num. Sim. 75, 14-21 (2019).

[27] O. Sultanov, "Stability and asymptotic analysis of the autoresonant capture in oscillating systems with combined excitation", SIAM J. Appl. Math. 78, 3103-3118 (2018).

[28] O. A. Sultanov, "Bifurcations of autoresonant modes in oscillating systems with combined excitation", Stud. Appl. Math. 144, 213-241 (2020).

[29] A. N. Kuznetsov, "Existence of solutions entering at a singular point of an autonomous system having a formal solution", Funct. Anal. Appl. 23, 308-317 (1989). 
[30] V. V. Kozlov and S. D. Furta, Asymptotic solutions of strongly nonlinear systems of differential equations. (Springer, Heidelberg, New York, Dordrecht, London, 2013).

[31] L. A. Kalyakin, "Existence theorems and estimates of solutions for equations of principal resonance", J. Math. Sci. 200, 82-95 (2014).

[32] H. Thieme, "Asymptotically autonomous differential equations in the plane", Rocky Mountain J. Math., 24, 351-380 (1994).

[33] O.A. Sultanov, "Stability and bifurcation phenomena in asymptotically Hamiltonian systems", arXiv: $2006.12957(2020)$.

[34] H. K. Khalil, Nonlinear systems. (Prentice Hall, Upper Saddle River, NJ, 2002). 